\title{
Localisation in a Growth Model with Interaction
}

\author{
M. Costa ${ }^{1}$ - M. Menshikov ${ }^{1}$ - V. Shcherbakov ${ }^{2}$. \\ M. Vachkovskaia ${ }^{3}$
}

Received: 3 January 2018 / Accepted: 2 May 2018 / Published online: 8 May 2018

(C) The Author(s) 2018

\begin{abstract}
This paper concerns the long term behaviour of a growth model describing a random sequential allocation of particles on a finite cycle graph. The model can be regarded as a reinforced urn model with graph-based interaction. It is motivated by cooperative sequential adsorption, where adsorption rates at a site depend on the configuration of existing particles in the neighbourhood of that site. Our main result is that, with probability one, the growth process will eventually localise either at a single site, or at a pair of neighbouring sites.
\end{abstract}

Keywords Growth process · Urn models · Cooperative sequential adsorption · Graph based interaction

Mathematics Subject Classification $60 \mathrm{~K} 35 \cdot 60 \mathrm{~J} 10$

\section{Introduction}

This paper concerns a probabilistic model describing a sequential allocation of particles on a finite cycle graph. The model is motivated by cooperative sequential adsorption (CSA) (see $[7,8]$ and references therein). CSA models are widely applied in physical chemistry

\footnotetext{
V. Shcherbakov

vadim.shcherbakov@rhul.ac.uk

M. Costa

m.r.costa@durham.ac.uk

M. Menshikov

mikhail.menshikov@durham.ac.uk

M. Vachkovskaia

marinav@ime.unicamp.br

1 Durham University, Durham, UK

2 Royal Holloway University of London, Surrey, UK

3 University of Campinas, Campinas, Brazil 


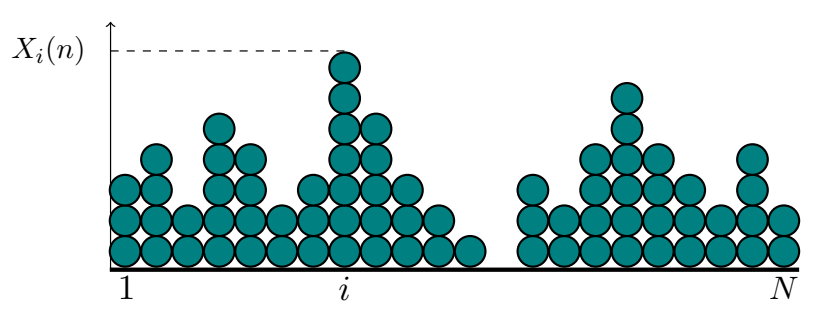

Fig. 1 Multilayer adsorption/random deposition model

for modelling adsorption processes on a material surface onto which particles are deposited at random. The main peculiarity of adsorption processes is that deposited particles change adsorption properties of the material. This motivates the growth rates defined in Eq. (1). The growth rates model a particular situation where the subsequent particles are more likely to be adsorbed around previously deposited particles.

There is typically a hard-core constraint associated with CSA. That is, the adsorption (growth) rate is zero at any location with more than a certain number of particles. The asymptotic shape of the spatial configuration of deposited particles is of primary interest in such models. Many probabilistic models of spatial growth by monolayer deposition, diffusion and aggregation dynamics present this characteristic. For instance, the Eden model [6], diffusion-limited aggregation process [22], first-passage percolation models [17] and contact interaction processes [18].

In contrast, in our model (defined in Sect. 2) we allow any number of particles to be deposited at each site. This is motivated by growing interfaces (Fig. 1) associated with multilayer adsorption processes (see $[2,10,15]$ ). Even though the random nature of these processes is usually emphasized in the physical literature, there is a limited number of rigorous formulations and published results in this field (most of them in $[14,16])$. Our model is closely related to a variant of random deposition models, but as we do not apply any of the techniques from this field, we refer the reader to the survey on surface growth [1].

Our model can be naturally interpreted in terms of interacting urn models. In the case of no interaction, in which the growth rate at site $i$ is given by $\Gamma\left(x_{i}\right)$, where $x_{i}$ is the number of existing particles at site $i$ and $\Gamma: \mathbb{Z}_{+} \rightarrow(0, \infty)$ is a given function (called the reinforcement rule [4] or feedback function [12]), our model coincides with a generalised Pólya urn (GPU) model with a particular reinforcement rule $\Gamma$. Each site (with no underlying graph structure) corresponds to a different colour of ball. The growth rule corresponds to choosing an existing ball of colour $i$, with probability proportional to $\Gamma\left(x_{i}\right)$, and adding a new ball of that colour. The case $\Gamma(x)=x$ is the classical Pólya urn.

The so called Rubin's exponential embedding (first appearing in [5]) classifies the two possible limiting behaviours in the above class of GPU models. Firstly, there almost surely exists a site $i$ that gets all but finitely many particles. Secondly, the number of particles at every site grows almost surely to infinity. For a comprehensive survey on urn models and their applications, see [13] and references therein.

In contrast, we consider growth rules with graph-based interactions (as in [19]) where the underlying graph is a cycle with $N$ sites. In our growth model the rate of growth at site $i$ is given by a site-dependent reinforcement rule $\Gamma_{i}=\exp \left(\lambda_{i} u_{i}\right)$, where $\lambda_{i}>0$ and $u_{i}$ is the number of existing particles in a neighbourhood of site $i$. This allows one to take into account the case where different sites might possibly have different reinforcement schemes (Fig. 2). In other words, the case where each site has its own intrinsic 'capacity' parameter, which 


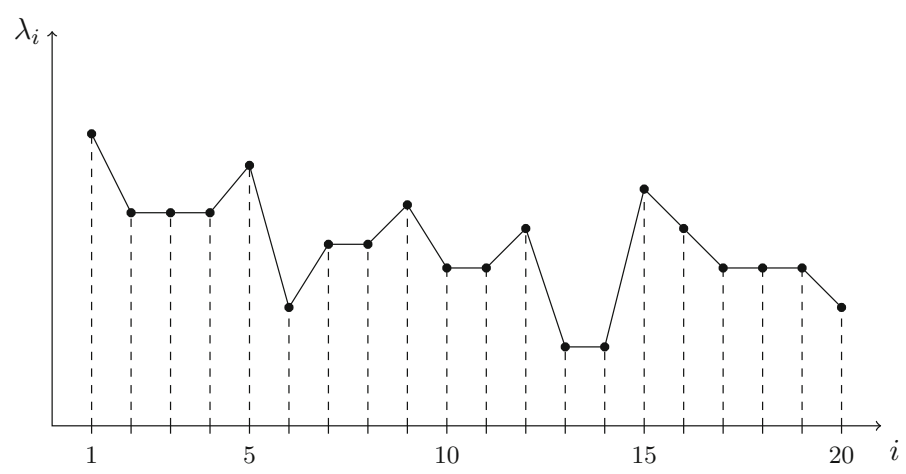

Fig. 2 An interpolated graph of a particular parameter set $\left(\lambda_{i}\right)_{i=1}^{20}$

is what would be expected in many real-life situations. Although the model can easily be defined for a general graph, the results will heavily depend on its topological properties. In this paper we only address the case of a cycle graph. See [3,9] for results on general graphs but different growth rules.

The main result of the present paper classifies, in terms of the set of parameters $\Lambda=$ $\left(\lambda_{i}\right)_{i=1}^{N}$, the two possible behaviours of the model. The first behaviour is localization of growth at a single site. This means that from a random moment of time onwards, all subsequent particles are allocated at a particular site. The second is localization of growth at a pair of neighbouring sites with equal $\lambda$ parameter. Similarly as in the first case, this means that from a random moment of time onwards, all subsequent particles are allocated at a particular pair of neighbouring sites. In particular, if $\lambda_{i} \neq \lambda_{i+1}$ for all $i$, then, with probability one, the growth will eventually localise at a single site. On the other hand, if $\lambda_{i} \equiv \lambda$, then, with probability one, the growth will eventually localise at a pair of neighbouring sites. In the general case of a fixed and arbitrary parameter set $\Lambda$, only the above two types of limiting behaviour are possible. Theorem 1 below provides a complete characterization of the parameter set $\Lambda$ and associated subsets where only one of the regimes, or both, may happen.

The model with $\Gamma_{i}=\exp \left(\lambda u_{i}\right)$, i.e., $\lambda_{i} \equiv \lambda \in \mathbb{R}$, was first considered in [19], and an analogue of Theorem 1 (Theorem 3 in [19]) was proved for this particular case of siteindependent parameter $\lambda$.

The paper is organised as follows. In Sect. 2, we formally define the model, fix some terminology and state Theorem 1 which is our main result. The proof of the theorem appears in Sect. 6 and relies essentially on Lemmas 1-8 stated in Sect. 3 and proved in Sect. 5. Section 4 contains results concerning sums of random geometric progressions, which are of interest in their own right. These results combined with stochastic domination techniques are constantly used in the proofs of Lemmas 5-8.

\section{The Model and Main Result}

Consider a cycle graph with $N \geq 4$ vertices (sites) enumerated by the first $N$ natural numbers such that $1 \sim 2 \sim \ldots \sim N-1 \sim N \sim 1$, where $i \sim j$ indicates that sites $i$ and $j$ are incident. Let $\mathbb{Z}_{+}$be the set of non-negative integers and $\Lambda=\left\{\lambda_{1}, \ldots, \lambda_{N}\right\}$ be an arbitrary 
set of positive real numbers. Given $\mathbf{x}=\left(x_{1}, \ldots, x_{N}\right) \in \mathbb{Z}_{+}^{N}$, define the growth rates as

$$
\Gamma_{i}(\mathbf{x})=e^{\lambda_{i}\left(x_{i}+\sum_{j \sim i} x_{j}\right)}, \quad i=1, \ldots, N .
$$

Consider a discrete-time Markov chain $X(n)=\left(X_{1}(n), \ldots, X_{N}(n)\right) \in \mathbb{Z}_{+}^{N}$ with the following transition probabilities

$$
\mathrm{P}\left(X_{i}(n+1)=X_{i}(n)+1 \mid X(n)=\mathbf{x}\right)=\frac{\Gamma_{i}(\mathbf{x})}{\sum_{k=1}^{N} \Gamma_{k}(\mathbf{x})}, \quad i=1, \ldots, N, \quad \mathbf{x} \in \mathbb{Z}_{+}^{N} .
$$

The Markov chain describes the evolution of the number of particles sequentially allocated at each site of the graph. Given the configuration of particles $X(n)=\mathbf{x} \in \mathbb{Z}_{+}^{N}$ at time $n$, the next incoming particle is placed at site $i$ with probability proportional to $\Gamma_{i}(\mathbf{x})$.

Definition 1 For $i \in\{1, \ldots, N\}(\operatorname{modulo} N)$

(1) a site $\{i\}$ is a local minimum, if $\lambda_{i}<\min \left(\lambda_{i-1}, \lambda_{i+1}\right)$;

(2) a pair of sites $\{i, i+1\}$ is a local minimum of size 2 , if $\lambda_{i}=\lambda_{i+1}<\min \left(\lambda_{i-1}, \lambda_{i+2}\right)$;

(3) a site $\{i\}$ is a local maximum, if $\lambda_{i}>\max \left(\lambda_{i-1}, \lambda_{i+1}\right)$;

(4) a pair of sites $\{i, i+1\}$ is a saddle point, if

$$
\min \left(\lambda_{i-1}, \lambda_{i+2}\right)<\lambda_{i}=\lambda_{i+1}<\max \left(\lambda_{i-1}, \lambda_{i+2}\right) ;
$$

(5) a site $\{i\}$ is a growth point, if either $\lambda_{i-1}<\lambda_{i}<\lambda_{i+1}$, or $\lambda_{i-1}>\lambda_{i}>\lambda_{i+1}$.

Definition 2 Let $\{i, i+1\}$ be a local minimum of size two. We say that it is a local minimum of size 2 and

(1) type 1 , if $\lambda_{i}=\lambda_{i+1}>\frac{\lambda_{i-1} \lambda_{i+2}}{\lambda_{i-1}+\lambda_{i+2}}$,

(2) type 2 , if $\lambda_{i}=\lambda_{i+1} \leq \frac{\lambda_{i-1} \lambda_{i+2}}{\lambda_{i-1}+\lambda_{i+2}}$.

The following theorem is the main result of the paper.

Theorem 1 For every $X(0)=\mathbf{x} \in \mathbb{Z}_{+}^{N}$ and

(i) for every local maximum $\{k\}$, with positive probability,

$$
\lim _{n \rightarrow \infty} X_{i}(n)=\infty \text { if and only if } i=k \text {; }
$$

(ii) for every pair $\{k, k+1\}$ where $\lambda_{k}=\lambda_{k+1}=: \lambda$, but not a local minimum of size 2 and type 2 , with positive probability,

$$
\begin{aligned}
& \lim _{n \rightarrow \infty} X_{i}(n)=\infty, \quad \text { if and only if } i \in\{k, k+1\}, \text { and } \\
& \lim _{n \rightarrow \infty} \frac{X_{k+1}(n)}{X_{k}(n)}=e^{\lambda R},
\end{aligned}
$$

where $R=\lim _{n \rightarrow \infty}\left[X_{k+2}(n)-X_{k-1}(n)\right] \in \mathbb{Z}$.

No other limiting behaviour is possible. That is, with probability 1, exactly one of the above events occurs in a random location $\{k\}$ or $\{k, k+1\}$ as described in (i) and (ii), respectively. 


\section{Lemmas}

We start with notations that will be used throughout the proofs. Given $i=1, \ldots, N$, define the following events

$$
\begin{aligned}
& A_{n}^{i}:=\{\text { at time } n \text { a particle is placed at site } i\}, \quad n \in \mathbb{Z}_{+}, \\
& A_{n}^{i, i+1}:=A_{n}^{i} \cup A_{n}^{i+1}, \quad n \in \mathbb{Z}_{+} .
\end{aligned}
$$

Define also the following events

$$
\begin{aligned}
& A_{\left[n_{1}, n_{2}\right]}^{i}:=\bigcap_{n=n_{1}}^{n_{2}} A_{n}^{i}, \\
& A_{\left[n_{1}, n_{2}\right]}^{i, i+1}:=\bigcap_{n=n_{1}}^{n_{2}} A_{n}^{i, i+1},
\end{aligned}
$$

indicating that from time $n_{1}$ to $n_{2}$ all particles are placed at site $i$, and at sites $i$ or $i+1$, respectively. Further, events $A_{[n, \infty)}^{i}$ and $A_{[n, \infty)}^{i, i+1}$ denote the corresponding limiting cases as $n_{2}$ goes to infinity.

Let $\mathbf{e}_{i} \in \mathbb{Z}_{+}^{N}$ be a vector, whose $i$ th coordinate is 1 , and all other coordinates are zero. Given $\mathbf{x} \in \mathbb{Z}_{+}^{N}$, define the following probability measure $\mathrm{P}_{\mathbf{x}}(\cdot)=\mathrm{P}(\cdot \mid X(0)=\mathbf{x})$.

Remark 1 In lemmas and proofs below we denote by $\epsilon$ and $\varepsilon$, possibly with subscripts, various positive constants whose values do not depend on the starting configuration $\mathbf{x}$ and may vary from line to line. This is essential for the proof of Theorem 1. Also, the results are stated only for the essentially different cases, and whenever there are trivially symmetric situations (e.g., $\lambda_{k-1}<\lambda_{k}<\lambda_{k+1}$ and $\lambda_{k-1}>\lambda_{k}>\lambda_{k+1}$ ), we state and prove only one of them in order to avoid unnecessary repetition.

Lemma 1 Suppose that $\{k\}$ is a local maximum, and $\mathbf{x} \in \mathbb{Z}_{+}^{N}$ is such that $\Gamma_{k}(\mathbf{x})=$ $\max _{i} \Gamma_{i}(\mathbf{x})$. Then, with positive probability, all subsequent particles are allocated at $k$, i.e., $\mathrm{P}_{\mathbf{x}}\left(A_{[1, \infty)}^{k}\right) \geq \epsilon$ for some $\epsilon>0$.

Lemma 1 describes the only case where localisation of growth at a single site can occur, namely, at a local maximum.

Lemma 2 Suppose that $\{k\}$ is a growth point, and $\mathbf{x} \in \mathbb{Z}_{+}^{N}$ is such that $\Gamma_{k}(\mathbf{x})=\max _{i} \Gamma_{i}(\mathbf{x})$. If $\lambda_{k-1}<\lambda_{k}<\lambda_{k+1}$, then there exist $n=n(\mathbf{x}, \Lambda) \in \mathbb{Z}_{+}$and $\epsilon>0$, such that $\mathrm{P}_{\mathbf{x}}\left(A_{[1, n]}^{k}\right) \geq \epsilon$ and $\Gamma_{k+1}\left(\mathbf{x}+n \mathbf{e}_{k}\right)=\max _{i} \Gamma_{i}\left(\mathbf{x}+n \mathbf{e}_{k}\right)$.

Lemma 3 Suppose that $\{k\}$ is a localminimum, and $\mathbf{x} \in \mathbb{Z}_{+}^{N}$ is such that $\Gamma_{k}(\mathbf{x})=\max _{i} \Gamma_{i}(\mathbf{x})$. Then there exist $n=n(\mathbf{x}, \Lambda) \in \mathbb{Z}_{+}$and $\epsilon>0$, such that $\mathrm{P}_{\mathbf{x}}\left(A_{[1, n]}^{k}\right) \geq \epsilon$ and $\max \left(\Gamma_{k-1}(\mathbf{x}+\right.$ $\left.\left.n \mathbf{e}_{k}\right), \Gamma_{k+1}\left(\mathbf{x}+n \mathbf{e}_{k}\right)\right)=\max _{i} \Gamma_{i}\left(\mathbf{x}+n \mathbf{e}_{k}\right)$.

Lemmas 2-3 describe the following effect. If the maximal rate is attained at a site which is either a growth point or a local minimum, then, with positive probability, allocating $n=$ $n(\mathbf{x}, \Lambda)$ particles at that site results in relocation of the maximal rate to a nearest neighbour with larger parameter $\lambda$. The number of particles required for relocation (the relocation time) is deterministic and depends only on the starting configuration $\mathbf{x}$ and parameter set $\Lambda$.

Lemma 4 Suppose that $\Gamma_{k}(\mathbf{x})=\max _{i} \Gamma_{i}(\mathbf{x})$. 
(1) $\lambda_{k-1}<\lambda_{k}=\lambda_{k+1} \geq \lambda_{k+2}$; or

(2) $\lambda_{k-1}=\lambda_{k}=\lambda_{k+1} \geq \lambda_{k+2}$, and $\Gamma_{k+1}(\mathbf{x}) \geq \Gamma_{k-1}(\mathbf{x})$,

then, with positive probability, all subsequent particles are allocated at sites $\{k, k+1\}$, i.e., $\mathrm{P}_{\mathbf{x}}\left(A_{[1, \infty)}^{k, k+1}\right) \geq \epsilon$ for some $\epsilon>0$.

Lemma 4 describes a particular case that implies the second possible limiting behaviour of the model, i.e., localisation of growth at a pair of neighbouring sites.

Definition 3 Define the following stopping times

$$
\begin{aligned}
& \tau_{k}=\inf \left(n: X_{k}(n)=X_{k}(0)+1\right), \\
& w_{k}^{+}=\min \left(\tau_{i}: i \neq k, k+1, k+2\right), \quad \text { for } k=1, \ldots, N,
\end{aligned}
$$

where the usual convention is that

$$
\inf (\emptyset)=\infty \text { and } \min (a, \infty)=a, \quad \text { for } a \in \mathbb{R}_{+} \cup\{\infty\}
$$

The above stopping times and the quantities $r, z_{1}$ and $z_{2}$ below will appear throughout Lemmas 4-8 and their proofs.

Definition 4 Given $\mathbf{x} \in \mathbb{Z}_{+}^{N}$ define

$$
r:=r(\mathbf{x})=x_{k+2}-x_{k-1} .
$$

In addition, if a pair of sites $\{k, k+1\}$ is such that $\lambda_{k}=\lambda_{k+1}=: \lambda$ and

$$
\begin{aligned}
& \lambda_{k-1}>\lambda, \quad \text { define } z_{1}=\frac{1}{\lambda} \log \left(\frac{\lambda_{k-1}-\lambda}{\lambda}\right), \\
& \lambda_{k+2}>\lambda, \quad \text { define } z_{2}=\frac{1}{\lambda} \log \left(\frac{\lambda}{\lambda_{k+2}-\lambda}\right) .
\end{aligned}
$$

Before stating Lemma 5, let us denote by $B_{k}$ the event in which a particle arrives in finite time at $k+2$ before anywhere outside $\{k, k+1, k+2\}$. That is to say,

$$
B_{k}:=\left\{\tau_{k+2}<w_{k}^{+}\right\} \text {. }
$$

Lemma 5 Suppose that a pair of sites $\{k, k+1\}$ is a saddle point with $\lambda_{k-1}<\lambda_{k}=\lambda_{k+1}=$ : $\lambda<\lambda_{k+2}$, and $\mathbf{x} \in \mathbb{Z}_{+}^{N}$ is such that

$$
\max \left(\Gamma_{k}(\mathbf{x}), \Gamma_{k+1}(\mathbf{x})\right)=\max _{i} \Gamma_{i}(\mathbf{x}) .
$$

(1) Then there exists $\epsilon>0$ such that

$$
\mathrm{P}_{\mathbf{x}}\left(A_{[1, \infty)}^{k, k+1} \bigcup B_{k}\right)=\mathrm{P}_{\mathbf{x}}\left(A_{[1, \infty)}^{k, k+1}\right)+\mathrm{P}_{\mathbf{x}}\left(B_{k}\right) \geq \epsilon .
$$

(2) If $r<z_{2}$, then, with positive probability, all subsequent particles are allocated at sites $\{k, k+1\}$, i.e., $\mathrm{P}_{\mathbf{x}}\left(A_{[1, \infty)}^{k, k+1}\right) \geq \varepsilon$ for some $\varepsilon>0$.

(3) If $r \geq z_{2}$, then $\mathrm{P}_{\mathbf{x}}\left(A_{[1, \infty)}^{k, k+1}\right)=0$, and, hence, $\mathrm{P}_{\mathbf{x}}\left(B_{k}\right) \geq \epsilon$.

(4) If $r>z_{2}$ is strict, then, with positive probability, the maximal rate relocates as follows. There exists $\varepsilon>0$ such that

$$
\mathrm{P}_{\mathbf{x}}\left(B_{k}, \max _{i=k+2, k+3} \Gamma_{i}\left(X\left(\tau_{k+2}\right)\right)=\max _{i} \Gamma_{i}\left(X\left(\tau_{k+2}\right)\right)\right) \geq \varepsilon,
$$

where $\max _{i} \Gamma_{i}\left(X\left(\tau_{k+2}\right)\right)$ may be attained at $k+3$ only if $\lambda_{k+3}>\lambda$. 
Part (4) of Lemma 5 is similar to Lemmas 2-3 in that it also describes relocation of the maximal rate to a site with larger parameter $\lambda$. The main difference is that in Lemma 5 the relocation time is random. This is in contrast to Lemmas $2-3$, where the relocation time is deterministic.

The proposition and definition below are intended to clarify some assumptions and simplify some notations in Lemmas 6-8.

Proposition 1 Let $\{k, k+1\}$ be a local minimum of size 2 with $\lambda=\lambda_{k}=\lambda_{k+1}$, and let $r=r(\mathbf{x}), z_{1}$ and $z_{2}$ be quantities as in Definition 4. Then, $z_{1}<z_{2}$ if and only if local minimum $\{k, k+1\}$ is of type 1 , in which case there might exist $\mathbf{x}$ such that $z_{1}<r<z_{2}$. Otherwise, if a local minimum $\{k, k+1\}$ is of type 2 , then $z_{2} \leq z_{1}$, in which case $r \geq z_{2}$ or $r \leq z_{1}$ for all $\mathbf{x}$.

Definition 5 Recall that $\tau_{k}:=\inf \left(n: X_{k}(n)=X_{k}(0)+1\right)$ and let us further define the following stopping times

$$
\begin{aligned}
\sigma_{k} & =\min \left(\tau_{k-1}, \tau_{k+2}\right), \\
w_{k} & =\min \left(\tau_{i}: i \neq k \pm 1, k, k+2\right),
\end{aligned}
$$

and following events

$$
\begin{aligned}
& D_{k}=\left\{\sigma_{k}<w_{k}\right\}, \\
& D_{k}^{\prime}=\left\{\tau_{k-1}<\min \left(\tau_{k+2}, w_{k}\right)\right\}, \\
& D_{k}^{\prime \prime}=\left\{\tau_{k+2}<\min \left(\tau_{k-1}, w_{k}\right)\right\} .
\end{aligned}
$$

Note that $D_{k}^{\prime} \cap D_{k}^{\prime \prime}=\emptyset, D_{k}=D_{k}^{\prime} \cup D_{k}^{\prime \prime}$ and $A_{[1, \infty)}^{k, k+1} \cap D_{k}=\emptyset$.

Lemma 6 Suppose that $\{k, k+1\}$ is a local minimum of size 2 , and $\mathbf{x} \in \mathbb{Z}_{+}^{N}$ is such that $\max \left(\Gamma_{k}(\mathbf{x}), \Gamma_{k+1}(\mathbf{x})\right)=\max _{i} \Gamma_{i}(\mathbf{x})$.

(1) There exists $\epsilon>0$ such that

$$
\mathrm{P}_{\mathbf{x}}\left(A_{[1, \infty)}^{k, k+1} \bigcup D_{k}\right)=\mathrm{P}_{\mathbf{x}}\left(A_{[1, \infty)}^{k, k+1}\right)+\mathrm{P}_{\mathbf{x}}\left(D_{k}\right) \geq \epsilon .
$$

(2) If $z_{1}<r<z_{2}$ (only possible if $\{k, k+1\}$ is of type 1 ), then, with positive probability, all subsequent particles are allocated at sites $\{k, k+1\}$, i.e., $\mathrm{P}_{\mathbf{x}}\left(A_{[1, \infty)}^{k, k+1}\right)>\varepsilon$ for some $\varepsilon>0$.

(3) If $r \leq z_{1}$ or $r \geq z_{2}$ (always the case if $\{k, k+1\}$ is of type 2 ), then $\mathrm{P}_{\mathbf{x}}\left(A_{[1, \infty)}^{k, k+1}\right)=0$ and, hence, $\mathrm{P}_{\mathbf{x}}\left(D_{k}\right) \geq \epsilon$.

Lemma 6 is analogous to Parts (1)-(3) of Lemma 5 for the case of a local minimum of size 2. An analogue of Part (4) of Lemma 5 in the same situation is provided by Lemma 7.

Lemma 7 Suppose that local minimum $\{k, k+1\}$ is of size 2 with $\lambda_{k}=\lambda_{k+1}:=\lambda$, and $\mathbf{x} \in \mathbb{Z}_{+}^{N}$ is such that $\max \left(\Gamma_{k}(\mathbf{x}), \Gamma_{k+1}(\mathbf{x})\right)=\max _{i} \Gamma_{i}(\mathbf{x})$.

(1) If $\{k, k+1\}$ is of type 1 and $r<z_{1}$, or $\{k, k+1\}$ is of type 2 and $r<z_{2}$ then

$$
\mathrm{P}_{\mathbf{x}}\left(D_{k}^{\prime}, \max _{i=k-2, k-1} \Gamma_{i}\left(X\left(\tau_{k-1}\right)\right)=\max _{i=1, \ldots, N} \Gamma_{i}\left(X\left(\tau_{k-1}\right)\right)\right) \geq \varepsilon,
$$

for some $\varepsilon>0$, where $\max _{i} \Gamma_{i}\left(X\left(\tau_{k-1}\right)\right)$ may be attained at $k-2$ only if $\lambda_{k-2}>\lambda$. 
(2) If $\{k, k+1\}$ is of type 1 and $r>z_{2}$, or $\{k, k+1\}$ is of type 2 and $r>z_{1}$ then

$$
\mathrm{P}_{\mathbf{x}}\left(D_{k}^{\prime \prime}, \max _{i=k+2, k+3} \Gamma_{i}\left(X\left(\tau_{k+2}\right)\right)=\max _{i=1, \ldots, N} \Gamma_{i}\left(X\left(\tau_{k+2}\right)\right)\right) \geq \varepsilon,
$$

for some $\varepsilon>0$, where $\max _{i} \Gamma_{i}\left(X\left(\tau_{k+2}\right)\right)$ may be attained at $k+3$ only if $\lambda_{k+3}>\lambda$.

(3) If $\{k, k+1\}$ is of type 2 and $z_{2}<r<z_{1}$, then

$$
\begin{aligned}
& \mathrm{P}_{\mathbf{x}}\left(D_{k}^{\prime}, \max _{i=k-2, k-1} \Gamma_{i}\left(X\left(\tau_{k-1}\right)\right)=\max _{i=1, \ldots, N} \Gamma_{i}\left(X\left(\tau_{k-1}\right)\right)\right) \\
& \quad+\mathrm{P}_{\mathbf{x}}\left(D_{k}^{\prime \prime}, \max _{i=k+2, k+3} \Gamma_{i}\left(X\left(\tau_{k+2}\right)\right)=\max _{i=1, \ldots, N} \Gamma_{i}\left(X\left(\tau_{k+2}\right)\right)\right) \geq \varepsilon,
\end{aligned}
$$

for some $\varepsilon>0$, where $\max \Gamma_{i}$ follows the corresponding prescriptions as above.

Remark 2 The next lemma concerns the borderline cases in between having a local minimum $\{k, k+1\}$ of size 2 and type 1 or a saddle point. For example, in notations of Lemma 7 these cases are formally obtained by setting either $\lambda_{k-1}=\lambda$ (where $-\infty=z_{1}<z_{2}$ ), or $\lambda_{k+2}=\lambda$ (where $z_{1}<z_{2}=\infty$ ). As both cases can be addressed in similar ways, the lemma below deals only with the case $\lambda_{k-1}=\lambda$.

Lemma 8 Suppose that sites $\{k-1, k, k+1, k+2\}$ are such that

$$
\lambda_{k-1}=\lambda_{k}=\lambda_{k+1}=: \lambda<\lambda_{k+2},
$$

$\mathbf{x} \in \mathbb{Z}_{+}^{N}$ is such that $\max \left(\Gamma_{k}(\mathbf{x}), \Gamma_{k+1}(\mathbf{x})\right)=\max _{i} \Gamma_{i}(\mathbf{x})$ and, additionally, $\Gamma_{k-1}(\mathbf{x}) \leq$ $\Gamma_{k+1}(\mathbf{x})$.

(1) There exists $\epsilon>0$ such that

$$
\mathrm{P}_{\mathbf{x}}\left(A_{[1, \infty)}^{k, k+1} \bigcup D_{k}\right)=\mathrm{P}_{\mathbf{x}}\left(A_{[1, \infty)}^{k, k+1}\right)+\mathrm{P}_{\mathbf{x}}\left(D_{k}\right) \geq \epsilon .
$$

(2) If $r<z_{2}$, then, with positive probability all subsequent particles are allocated at sites

$\{k, k+1\}$, i.e., $\mathrm{P}_{\mathbf{x}}\left(A_{[1, \infty)}^{k, k+1}\right) \geq \epsilon$ for some $\varepsilon>0$.

(3) If $r \geq z_{2}$, then $\mathrm{P}_{\mathbf{x}}\left(A_{[1, \infty)}^{k, k+1}\right)=0$ and, hence, $\mathrm{P}_{\mathbf{x}}\left(D_{k}\right) \geq \epsilon$.

(4) If $r>z_{2}$, then there exists $\varepsilon>0$ such that

$$
\mathrm{P}_{\mathbf{x}}\left(B_{k}, \max _{i=k+2, k+3} \Gamma_{i}\left(X\left(\tau_{k+2}\right)\right)=\max _{i} \Gamma_{i}\left(X\left(\tau_{k+2}\right)\right)\right) \geq \varepsilon,
$$

where $\max _{i} \Gamma_{i}\left(X\left(\tau_{k+2}\right)\right)$ may be attained at $k+3$ only if $\lambda_{k+3}>\lambda$.

The following corollary concerns those cases covered by Parts (3) of Lemmas 5, 6 and 8, where the configuration parameter $r$ is equal to one of the model parameters $z_{1}$ and $z_{2}$. In what follows we call them critical cases.

Corollary 1 For the critical cases, relocation of the maximal rate to a site with larger parameter $\lambda$ also occurs, with positive probability, in finite time.

Remark 3 Let us remark the following.

(1) It is important to emphasize that in all the above cases where the maximal rate $\max _{i} \Gamma_{i}(\mathbf{x})$ eventually relocates with positive probability, it always relocates to a site with strictly larger parameter $\lambda$.

(2) Note that Lemmas 2, 3, 5 and 7 can be appropriately reformulated in order to cover the symmetric cases by simply re-labelling the graph sites in reverse order as the graph is a cycle. For example, if $\{k, k+1\}$ is a saddle point as in Lemma 5, then the corresponding symmetric case would be $\lambda_{k-1}>\lambda_{k}=\lambda_{k+1}>\lambda_{k+2}$, etc. 


\section{Random Geometric Progressions and Bernoulli Measures}

The statements and propositions in this section are essential building blocks for the proof of lemmas which follow. The reason is that along the proofs of Lemmas 4-8 we need to analyse the limiting behaviour of random variables of the form $\sum_{i=0}^{n} \prod_{j=1}^{i} \zeta_{j}$, as $n \rightarrow \infty$, where $\left\{\zeta_{j}, j \geq 1\right\}$ is an i.i.d. sequence of positive random variables. It will also be necessary to compare such variables and introduce some stochastic domination concepts to enable us to carry out uniform estimates not depending on the starting configuration $X(0)=\mathbf{x}$. We refer to [21] for standard definitions and basic properties of stochastic domination. The following notations are used throughout. Given random variables $X$ and $Y$ (or sequences $X$ and $Y$ ), we write $X \geq_{s t} Y$ if $X$ stochastically dominates $Y$. Similarly, given two probability measures $v$ and $\mu$, we write $\mu \geq_{s t} v$ if $\mu$ stochastically dominates $v$.

Random geometric progressions In this subsection we consider random variables realised on a certain probability space $(\Omega, \mathcal{F}, \mathrm{P})$. E denotes the expectation with respect to probability measure P. If $X$ and $Y$ are random variables or sequences such that $X \geq_{s t} Y$, then we may assume that $\mathrm{P}$ is a coupling of probability distributions of $X$ and $Y$ such that $\mathrm{P}(X \geq Y)=1$. Such a coupling exists by Strassen's theorem ([20]).

Given a random sequence $\zeta=\left\{\zeta_{i}, i \geq 1\right\}$, define

$$
Y_{i}(\zeta)=\prod_{j=1}^{i} \zeta_{j}, \quad i \geq 1, \quad Y_{0}(\zeta)=1, \quad \text { and } \quad Z_{n}(\zeta)=\sum_{i=0}^{n} Y_{i}(\zeta), \quad n \geq 1
$$

and

$$
Z(\zeta)=\sum_{i=0}^{\infty} Y_{i}(\zeta)
$$

Proposition 2 (1) Let $\zeta=\left\{\zeta_{i}, i \geq 1\right\}$ be an i.i.d. sequence of positive random variables such that $\mathrm{E}\left(\log \left(\zeta_{i}\right)\right)<0$. Then $\mathrm{P}(Z(\zeta)<\infty)=1$ and, consequently, $\mathrm{E}\left(e^{-Z(\zeta)}\right)>0$.

(2) Let $\theta=\left\{\theta_{i}, i \geq 1\right\}$ be another i.i.d. sequence of positive random variables such that $\mathrm{E}\left(\log \left(\theta_{i}\right)\right)<0$ and $\theta \geq$ st $\zeta$. Then $\mathrm{E}\left(e^{-Z(\zeta)}\right) \geq \mathrm{E}\left(e^{-Z(\theta)}\right)$.

Proof of Proposition 2 The first statement of the proposition is a well known simple fact in the theory of random walk in a random environment. Indeed, denote $\mathrm{E}\left(\log \left(\zeta_{i}\right)\right)=a<0$. Given $\delta>0$ such that $a+\delta<0$, it follows from the strong law of large numbers that $Y_{n}<e^{(a+\delta) n}$ for all but finitely many $n$ almost surely. Therefore, a tail of $Z(\zeta)$ is eventually majorised by the corresponding tail of a converging geometric progression. In turn, finiteness of $Z(\zeta)$ implies positiveness of the expectation. Moreover, note that $e^{Z(\cdot)}$ is an increasing function. Therefore, $e^{-Z(\zeta)} \geq_{s t} e^{-Z(\theta)}$ and hence, $\mathrm{E}\left(e^{-Z(\zeta)}\right) \geq \mathrm{E}\left(e^{-Z(\theta)}\right)$ as claimed.

Definition 6 Let $\zeta=\left\{\zeta_{i}, i \geq 1\right\}$ and $\eta=\left\{\eta_{j}, j \geq 1\right\}$ be i.i.d. sequences of positive random variables. Sequence $\eta$ is said to be reciprocal to $\zeta$ if $\eta_{1}$ has the same distribution as $1 / \zeta_{1}$.

The following proposition follows from basic properties of stochastic domination.

Proposition 3 Let $X$ and $Y$ be two i.i.d. sequences of positive random variables, and let $\eta_{X}$ and $\eta_{Y}$ be their corresponding reciprocal sequences. If $X \geq_{s t} Y$ then $\eta_{X} \leq_{s t} \eta_{Y}$.

Proposition 4 Let $\zeta=\left\{\zeta_{i}, i \geq 1\right\}$ be an i.i.d. sequence of positive random variables such that $\mathrm{E}\left(\log \left(\zeta_{i}\right)\right)>0$. Let $\left\{Y_{i}, i \geq 0\right\}$ and $\left\{Z_{n}(\zeta), n \geq 1\right\}$ be the random variables as in (8). 
Define the following random sequence

$$
F_{n}(\zeta)=Z_{n}(\zeta) / Y_{n}(\zeta), \quad n \geq 1
$$

Then, $F_{n}(\zeta)$ converges in distribution to

$$
Z(\eta)=1+\sum_{i=1}^{\infty} \prod_{j=1}^{i} \eta_{j}, \quad \text { as } n \rightarrow \infty,
$$

where $\eta$ is the sequence reciprocal to $\zeta$. Moreover, $Z(\eta)$ is almost surely finite and $Z(\eta) \geq_{s t}$ $F_{n}(\zeta)$ for any $n \geq 1$.

Proof of Proposition 4 First, note that for every $n \geq 1$,

$$
F_{n}(\zeta)=1+\sum_{i=1}^{n} \prod_{j=1}^{i} \zeta_{n-j+1}^{-1}=1+\sum_{i=1}^{n} \prod_{j=1}^{i} \eta_{j}^{(n)},
$$

where $\eta_{j}^{(n)}=\zeta_{n-j+1}^{-1}$. This means that $F_{n}(\zeta)$ has the same distribution as $Z_{n}(\eta)$ defined for the sequence $\eta=\left\{\eta_{i}, i \geq 1\right\}$ reciprocal to $\zeta$. Therefore, $F_{n}(\zeta)$ converges in distribution to $Z(\eta)$. In addition, $\mathrm{E}\left(\log \left(\eta_{1}\right)\right)=-\mathrm{E}\left(\log \left(\zeta_{1}\right)\right)<0$. Therefore, by Proposition $2, Z(\eta)$ is almost surely finite. Finally, it follows by construction that $Z(\eta) \geq_{s t} F_{n}(\zeta), n \geq 1$.

Proposition 5 Let $\zeta=\left\{\zeta_{i}, i \geq 1\right\}$ be an i.i.d. sequence of positive random variables such that $\mathrm{E}\left(\log \left(\zeta_{i}\right)\right)=a>0$, and $\eta=\left\{\eta_{i}, i \geq 1\right\}$ be its reciprocal sequence. Given $0<\gamma<1$, define the following stopping time

$$
\widehat{m}=\min \left(n: \gamma Y_{n}(\zeta) \geq 1\right) .
$$

Then both $Z(\eta)<\infty$ and $Z_{\widehat{m}-1}(\zeta)<\infty$ almost surely, $\gamma Z_{\widehat{m}-1}(\zeta) \leq_{s t} Z(\eta)$, and, hence,

$$
\mathrm{E}\left(e^{-\gamma Z_{\widehat{m}-1}(\zeta)}\right) \geq \mathrm{E}\left(e^{-Z(\eta)}\right)>0
$$

Proof of Proposition 5 By Proposition 4, $Z(\eta)$ is almost surely finite and $F_{n}(\zeta) \leq_{s t} Z(\eta)$ for all $n \geq 1$. Therefore, $F_{\widehat{m}-1}(\zeta) \leq_{s t} Z(\eta)$. Since $\gamma Y_{\widehat{m}-1}(\zeta)<1$ we obtain that

$$
\gamma Z_{\widehat{m}-1}(\zeta)<Z_{\widehat{m}-1}(\zeta) / Y_{\widehat{m}-1}(\zeta)=F_{\widehat{m}-1}(\zeta)
$$

Consequently, $\gamma Z_{\widehat{m}-1}(\zeta) \leq_{s t} Z(\eta)$, which implies (10) as claimed.

Proposition 6 Let $\zeta=\left(\zeta_{i}, i \geq 1\right)$ and $\theta=\left(\theta_{i}, i \geq 1\right)$ be i.i.d. sequences of positive random variables such that $\mathrm{E}\left(\log \left(\theta_{1}\right)\right)>0$ and $\zeta_{1} \geq_{s t} \theta_{1}$. Let $\eta_{\zeta}$ and $\eta_{\theta}$ be sequences reciprocal to $\zeta$ and $\theta$, respectively. Given $0<\gamma<1$, let $\widehat{m}$ be the stopping time for sequence $\zeta$ as in (9). Then

$$
\mathrm{E}\left(e^{-\gamma Z_{\widehat{m}-1}(\zeta)}\right) \geq \mathrm{E}\left(e^{-Z\left(\eta_{\theta}\right)}\right)
$$

Proof of Proposition 6 Note that $\zeta_{1} \geq_{s t} \theta_{1}$ implies $\mathrm{E}\left(\log \left(\zeta_{1}\right)\right)>0$. By Proposition 4 both $Z\left(\eta_{\zeta}\right)$ and $Z\left(\eta_{\theta}\right)$ are almost surely finite. Further, by Proposition $3 \eta_{\zeta} \leq_{s t} \eta_{\theta}$. Therefore

$$
\mathrm{E}\left(e^{-Z\left(\eta_{\zeta}\right)}\right) \geq \mathrm{E}\left(e^{-Z\left(\eta_{\theta}\right)}\right) .
$$

By Proposition 5, it follows that

$$
\mathrm{E}\left(e^{-\gamma Z_{\widehat{m}-1}(\zeta)}\right) \geq \mathrm{E}\left(e^{-Z\left(\eta_{\zeta}\right)}\right) \geq \mathrm{E}\left(e^{-Z\left(\eta_{\theta}\right)}\right)
$$

as claimed. 
Bernoulli measures Now, we introduce a family of Bernoulli measures and some notations that will be used throughout proofs of Lemmas 4-8.

Let $\xi=\left(\xi_{i}, i \geq 1\right)$ be a sequence of independent Bernoulli random variables with success probability $p$. Let $\mu_{p}$ be the distribution of $\xi$, that is, the product Bernoulli measure defined on the set of infinite binary sequences, and denote by $\mathbb{E}_{p}$ the expectation with respect to the Bernoulli measure $\mu_{p}$.

Define

$$
U_{i}=\xi_{1}+\cdots+\xi_{i}, \quad i \geq 1,
$$

the binomial random variables corresponding to a Bernoulli sequence $\xi$.

Let $\lambda_{k-1}, \lambda_{k}, \lambda_{k+1}$ and $\lambda_{k+2}$ be $\lambda$-parameters corresponding to quadruples $\{k-1, k, k+$ $1, k+2\}$ of the graph sites such that $\lambda=\lambda_{k}=\lambda_{k+1}$ as in Lemmas 4-8. Let us define the following i.i.d. sequences

$$
\begin{aligned}
& \zeta_{1}=\left(\zeta_{1, i}=e^{\lambda_{k-1}\left(1-\xi_{i}\right)-\lambda}, i \geq 1\right), \\
& \zeta_{2}=\left(\zeta_{2, i}=e^{\lambda_{k+2} \xi_{i}-\lambda}, i \geq 1\right) .
\end{aligned}
$$

It is a well known fact that if $0<p^{\prime} \leq p^{\prime \prime}<1$, then $\mu_{p^{\prime}} \leq_{s t} \mu_{p^{\prime \prime}}$. This fact yields the following proposition.

Proposition 7 Let $\zeta_{1}^{\prime}, \zeta_{2}^{\prime}$ and $\zeta_{1}^{\prime \prime}, \zeta_{2}^{\prime \prime}$ be sequences defined by (12) for Bernoulli sequences with success probabilities $p^{\prime}$ and $p^{\prime \prime}$, respectively. If $0<p^{\prime} \leq p^{\prime \prime}<1$, then $\zeta_{1}^{\prime} \geq_{s t} \zeta_{1}^{\prime \prime}$ and $\zeta_{2}^{\prime} \leq_{s t} \zeta_{2}^{\prime \prime}$

Note that variables $Z_{n}$ [defined in (8)] corresponding to sequences $\zeta_{1}$ and $\zeta_{2}$ can be expressed in terms of Binomial random variables (11) as follows

$$
Z_{n}\left(\zeta_{1}\right)=\sum_{i=0}^{n} e^{\lambda_{k-1}\left(i-U_{i}\right)-\lambda i} \quad \text { and } \quad Z_{n}\left(\zeta_{2}\right)=\sum_{i=0}^{n} e^{\lambda_{k+2} U_{i}-\lambda i} .
$$

It is useful to note that if $\lambda_{k-1}=\lambda_{k+2}=\lambda$, then the above expressions are

$$
Z_{n}\left(\zeta_{1}\right)=\sum_{i=0}^{n} e^{-\lambda U_{i}} \text { and } Z_{n}\left(\zeta_{2}\right)=\sum_{i=0}^{n} e^{\lambda\left(U_{i}-i\right)} .
$$

\section{Proofs of Lemmas}

In the following proofs we show the existence of positive real constants $C, c, \epsilon$ and $\varepsilon$, whose exact values are immaterial and may vary from line to line, but which do not depend on the starting configuration $X(0)=\mathbf{x}$. In order to avoid notational clutter we shall denote initial allocation rates $\Gamma_{i}(\mathbf{x})$ simply by $\Gamma_{i}$ for all $i$. Moreover, whenever we fix index $k \in$ $\{1, \ldots, N\}$ and consider indices in the neighbourhood of $k$, those indices should be interpreted as modulo $N$.

\subsection{Proofs of Lemmas 1-3}

For short, denote $B=\sum_{i \neq k, k \pm 1} \Gamma_{i}$ and $Z=\sum_{i=1}^{N} \Gamma_{i}$. By assumption, $\Gamma_{k}=\max _{i=1, \ldots, N} \Gamma_{i}$, then

$$
\frac{\Gamma_{k-1}}{\Gamma_{k}} \leq 1, \quad \frac{\Gamma_{k+1}}{\Gamma_{k}} \leq 1, \quad \Gamma_{k} \geq \frac{Z}{N} \quad \text { and } \quad \frac{Z-\Gamma_{k}}{Z} \leq \frac{(N-1)}{N}
$$


It follows from the last two inequalities that

$$
\frac{B}{\Gamma_{k}} \leq N-1
$$

Proof of Lemma 1 Recall that $\lambda_{k}>\max \left(\lambda_{k-1}, \lambda_{k+1}\right)$. We need to prove the existence of a positive number $\epsilon$ such that

$$
\mathbb{P}_{\mathbf{x}}\left(A_{[1, \infty)}^{k}\right)=\prod_{n=0}^{\infty} \frac{\Gamma_{k} e^{\lambda_{k} n}}{\Gamma_{k-1} e^{\lambda_{k-1} n}+\Gamma_{k} e^{\lambda_{k} n}+\Gamma_{k+1} e^{\lambda_{k+1} n}+B}>\epsilon,
$$

where $\epsilon>0$ depends only on $\lambda_{k-1}, \lambda_{k}, \lambda_{k+1}$ and $N$.

Indeed, rewriting the identity in (16) and applying bounds (14) and (15),

$$
\begin{aligned}
& \mathbb{P}_{\mathbf{x}}\left(A_{[1, \infty)}^{k}\right) \\
& \quad=\exp \left(-\sum_{n=0}^{\infty} \log \left(1+\frac{\Gamma_{k-1}}{\Gamma_{k}} e^{\left(\lambda_{k-1}-\lambda_{k}\right) n}+\frac{\Gamma_{k+1}}{\Gamma_{k}} e^{\left(\lambda_{k+1}-\lambda_{k}\right) n}+\frac{B}{\Gamma_{k}} e^{-\lambda_{k} n}\right)\right) \\
& \quad \geq \exp \left(-\sum_{n=0}^{\infty} \log \left(1+e^{\left(\lambda_{k-1}-\lambda_{k}\right) n}+e^{\left(\lambda_{k+1}-\lambda_{k}\right) n}+(N-1) e^{-\lambda_{k} n}\right)\right) \\
& \quad \geq \exp \left(-C \sum_{n=0}^{\infty}\left(e^{\left(\lambda_{k-1}-\lambda_{k}\right) n}+e^{\left(\lambda_{k+1}-\lambda_{k}\right) n}+(N-1) e^{-\lambda_{k} n}\right)\right)>\epsilon>0,
\end{aligned}
$$

since the series in the exponent above converges. It is not hard to see that in the last inequality, $\epsilon$ should depend only on $\lambda_{k-1}, \lambda_{k}, \lambda_{k+1}$ and $N$.

Proof of Lemma 2 Recall that $\lambda_{k-1}<\lambda_{k}<\lambda_{k+1}$. We need to prove the existence of a finite positive integer $\hat{n}$ and a positive number $\epsilon$ such that

$$
\Gamma_{k+1} e^{\lambda_{k+1} \hat{n}} \geq \Gamma_{k} e^{\lambda_{k} \hat{n}}>\max \left(\Gamma_{k-1} e^{\lambda_{k-1} \hat{n}}, \max _{i \neq k, k \pm 1} \Gamma_{i}\right)
$$

and

$$
\mathbb{P}_{\mathbf{x}}\left(A_{[1, \hat{n}]}^{k}\right)=\prod_{n=0}^{\hat{n}-1} \frac{\Gamma_{k} e^{\lambda_{k} n}}{\Gamma_{k-1} e^{\lambda_{k-1} n}+\Gamma_{k} e^{\lambda_{k} n}+\Gamma_{k+1} e^{\lambda_{k+1} n}+B}>\epsilon
$$

where $\epsilon>0$ depends only on $\lambda_{k-1}, \lambda_{k}, \lambda_{k+1}$ and $N$. Note that the sequence $e^{\left(\lambda_{k+1}-\lambda_{k}\right) n}, n \geq$ 0 is exponentially increasing, so there exists the minimal integer $\hat{n}$ such that

$$
e^{\left(\lambda_{k+1}-\lambda_{k}\right) \hat{n}} \geq \frac{\Gamma_{k}}{\Gamma_{k+1}}, \quad \text { that is, } \quad \frac{\Gamma_{k+1}\left(\mathbf{x}+\hat{n} \mathbf{e}_{k}\right)}{\Gamma_{k}\left(\mathbf{x}+\hat{n} \mathbf{e}_{k}\right)} \geq 1 .
$$

Then, it is easy to see that

$$
\frac{\Gamma_{k+1}}{\Gamma_{k}} \sum_{n=0}^{\hat{n}-1} e^{\left(\lambda_{k+1}-\lambda_{k}\right) n} \leq C_{1}<\infty
$$


where $C_{1}$ depends only on $\lambda_{k}$ and $\lambda_{k+1}$. Further, rewriting the identity in (17) and using bounds (14), (15) and (18), gives that

$$
\begin{aligned}
& \mathbb{P}_{\mathbf{x}}\left(A_{[1, \hat{n}]}^{k}\right) \\
& \quad=\exp \left(-\sum_{n=0}^{\hat{n}-1} \log \left(1+\frac{\Gamma_{k-1}}{\Gamma_{k}} e^{\left(\lambda_{k-1}-\lambda_{k}\right) n}+\frac{\Gamma_{k+1}}{\Gamma_{k}} e^{\left(\lambda_{k+1}-\lambda_{k}\right) n}+\frac{B}{\Gamma_{k}} e^{-\lambda_{k} n}\right)\right) \\
& \geq \exp \left(-\sum_{n=0}^{\hat{n}-1} \log \left(1+e^{\left(\lambda_{k-1}-\lambda_{k}\right) n}+\frac{\Gamma_{k+1}}{\Gamma_{k}} e^{\left(\lambda_{k+1}-\lambda_{k}\right) n}+(N-1) e^{-\lambda_{k} n}\right)\right) \\
& \quad \geq \exp \left(-C_{2} \sum_{n=0}^{\hat{n}-1}\left(e^{\left(\lambda_{k-1}-\lambda_{k}\right) n}+\frac{\Gamma_{k+1}}{\Gamma_{k}} e^{\left(\lambda_{k+1}-\lambda_{k}\right) n}+(N-1) e^{-\lambda_{k} n}\right)\right)>\epsilon,
\end{aligned}
$$

for some $\epsilon>0$.

Proof of Lemma 3 Recall that $\lambda_{k}<\min \left(\lambda_{k-1}, \lambda_{k+1}\right)$. As in the proof of Lemma 2, we need to show existence of a finite positive integer $\hat{n}$ and a positive $\epsilon$ such that

$$
\max \left(\Gamma_{k-1} e^{\lambda_{k-1} \hat{n}}, \Gamma_{k+1} e^{\lambda_{k+1} \hat{n}}\right) \geq \Gamma_{k} e^{\lambda_{k} \hat{n}} \geq \max _{i \neq k, k \pm 1} \Gamma_{i}
$$

and

$$
\mathbb{P}_{\mathbf{x}}\left(A_{[1, \hat{n}]}^{k}\right)=\prod_{n=0}^{\hat{n}-1} \frac{\Gamma_{k} e^{\lambda_{k} n}}{\Gamma_{k-1} e^{\lambda_{k-1} n}+\Gamma_{k} e^{\lambda_{k} n}+\Gamma_{k+1} e^{\lambda_{k+1} n}+B}>\epsilon,
$$

where $\epsilon>0$ depends only on $\lambda_{k-1}, \lambda_{k}, \lambda_{k+1}$ and $N$. This can be shown similar to the proof of Lemma 2, and we skip details.

\subsection{Proofs of Lemmas 4-8}

\subsubsection{Notations}

We start with some preliminary considerations and notations that will be used throughout the proofs of Lemmas 4-8.

Let $\{k, k+1\}$ be a pair of sites such that $\lambda_{k}=\lambda_{k+1}=\lambda$. If, as defined in Definition 2, $r=r(\mathbf{x})=x_{k+2}-x_{k-1}$, then $\frac{\Gamma_{k+1}(\mathbf{x})}{\Gamma_{k}(\mathbf{x})}=e^{\lambda r}$. Therefore, given that the next particle is allocated at either $k$ or $k+1$, the conditional $\mathbf{P}_{\mathbf{x}}$-probability to choose $k+1$ is equal to

$$
p:=p(r)=\frac{\Gamma_{k+1}(\mathbf{x})}{\Gamma_{k}(\mathbf{x})+\Gamma_{k+1}(\mathbf{x})}=\frac{e^{\lambda r}}{1+e^{\lambda r}} .
$$

We henceforth denote $q=1-p$. Furthermore, probability $p$ does not change by adding particles at sites $k$ and $k+1$ since configuration parameter $r$ remains constant.

Note that $p(z)$, considered as a function of $z \in \mathbb{R}$, is monotonically increasing. A direct computation gives that unique solutions of equations $\lambda_{k-1}-\lambda=p(z) \lambda_{k-1}$ and $\lambda_{k+2} p(z)=\lambda$ are quantities $z_{1}$ and $z_{2}$ [defined in (3)], respectively.

Let $S_{n}$ be the number of additional particles at site $k+1$ at time $n \geq 1$. Let $S_{0}=$ 0 and $s(n)=\left(s_{0}, s_{1}, \ldots, s_{n}\right)$ be a fixed trajectory of a finite random sequence $S(n)=$ $\left(S_{0}, S_{1}, \ldots, S_{n}\right)$. Note that, by construction, any trajectory $s(n)$ is a sequence of non-negative integers such that $s_{0}=0$ and $s_{i}-s_{i-1} \in\{0,1\}, i=1, \ldots, n$. 
For short, denote

$$
\begin{aligned}
& \Gamma_{i}=\Gamma_{i}(\mathbf{x}), \quad \widetilde{\Gamma}_{k}=\sum_{i \neq k, k \pm 1, k+2} \Gamma_{i}, \\
& \gamma_{k, 1}=\frac{\Gamma_{k-1}}{\Gamma_{k}+\Gamma_{k+1}}, \quad \gamma_{k, 2}=\frac{\Gamma_{k+2}}{\Gamma_{k}+\Gamma_{k+1}}, \quad \tilde{\gamma}_{k}=\frac{\widetilde{\Gamma}_{k}}{\Gamma_{k}+\Gamma_{k+1}} .
\end{aligned}
$$

In the rest of this section we are going to derive expressions for probabilities $\mathrm{P}_{\mathbf{x}}\left(A_{[1, n+1]}^{k, k+1}\right), n \geq$ 1 , in terms of expectations with respect to a Bernoulli product measure on $\{0,1\}^{\infty}$ with parameter $p$ defined in (19). These expressions allow one to obtain lower and upper bounds for the above probabilities. We start with the case of fixed $n$ and then extend it to the case where $n$ is a stopping time.

In the above notations

$$
\begin{aligned}
\mathrm{P}_{\mathbf{x}} & \left(A_{i+1}^{k, k+1}, S_{i+1}=s_{i+1} \mid A_{[1, i]}^{k, k+1}, S_{i}=s_{i}\right) \\
& =\frac{p^{s_{i+1}-s_{i}} q^{1-\left(s_{i+1}-s_{i}\right)}\left(\Gamma_{k}+\Gamma_{k+1}\right) e^{\lambda i}}{\left(\Gamma_{k}+\Gamma_{k+1}\right) e^{\lambda i}+\Gamma_{k-1} e^{\lambda_{k-1}\left(i-s_{i}\right)}+\Gamma_{k+2} e^{\lambda_{k+2} s_{i}}+\widetilde{\Gamma}_{k}} \\
& =\frac{p^{s_{i+1}-s_{i}} q^{1-\left(s_{i+1}-s_{i}\right)}}{1+\gamma_{k, 1} e^{\lambda_{k-1}\left(i-s_{i}\right)-\lambda i}+\gamma_{k, 2} e^{\lambda_{k+2} s_{i}-\lambda i}+\widetilde{\gamma}_{k} e^{-\lambda i}} .
\end{aligned}
$$

Then, given $n$ we obtain by repeated conditioning that

$$
\mathrm{P}_{\mathbf{x}}\left(A_{[1, n+1]}^{k, k+1}, S_{n+1}=s_{n+1}, \ldots, S_{1}=s_{1}\right)=p^{s_{n+1}} q^{n+1-s_{n+1}} W_{n}\left(s_{1}, \ldots, s_{n}\right),
$$

where

$$
W_{n}\left(s_{1}, \ldots, s_{n}\right)=\prod_{i=0}^{n} \frac{1}{1+\gamma_{k, 1} e^{\lambda_{k-1}\left(i-s_{i}\right)-\lambda i}+\gamma_{k, 2} e^{\lambda_{k+2} s_{i}-\lambda i}+\tilde{\gamma}_{k} e^{-\lambda i}} .
$$

Consequently, we get that

$$
\begin{aligned}
\mathrm{P}_{\mathbf{x}}\left(A_{[1, n+1]}^{k, k+1}\right) & =\sum_{s(n+1)} p^{s_{n+1}} q^{n+1-s_{n+1}} W_{n}\left(s_{1}, \ldots, s_{n}\right), \\
& =\sum_{s(n)}(p+q) p^{s_{n}} q^{n-s_{n}} W_{n}\left(s_{1}, \ldots, s_{n}\right), \\
& =\sum_{s(n)} p^{s_{n}} q^{n-s_{n}} W_{n}\left(s_{1}, \ldots, s_{n}\right),
\end{aligned}
$$

where the sum in the first line is over all possible trajectories $s(n+1)=\left(s_{1}, \ldots, s_{n+1}\right)$ of $S(n+1)=\left(S_{1}, \ldots, S_{n+1}\right)$ and the other two are over all possible trajectories $s(n)=$ $\left(s_{1}, \ldots, s_{n}\right)$ of $S(n)=\left(S_{1}, \ldots, S_{n}\right)$. Therefore, we arrive to the following equation

$$
\mathrm{P}_{\mathbf{x}}\left(A_{[1, n+1]}^{k, k+1}\right)=\mathbb{E}_{p}\left(W_{n}\left(U_{1}, \ldots, U_{n}\right)\right),
$$

where $\mathbb{E}_{p}$ is the expectation with respect to the Bernoulli measure $\mu_{p}$ defined in Sect. 4 and $U_{i}, i \geq 1$, are Binomial random variables defined in (11).

Further, assumptions of Lemmas 4-8 imply that $\frac{\Gamma_{i}}{\Gamma_{k}+\Gamma_{k+1}} \leq 1, i=1, \ldots, N$. Therefore, quantity $\tilde{\gamma}_{k}$ defined in (20) can be bounded as follows

$$
\tilde{\gamma}_{k} \leq(N-4) \text {. }
$$


Using bound (24) and inequality $\log (1+z) \leq z$ for all $z \geq 0$ we obtain that

$$
\begin{aligned}
W_{n}\left(s_{1}, \ldots, s_{n}\right) & \geq \prod_{i=0}^{n} \frac{1}{1+\gamma_{k, 1} e^{\lambda_{k-1}\left(i-s_{i}\right)-\lambda i}+\gamma_{k, 2} e^{\lambda_{k+2} s_{i}-\lambda i}+(N-4) e^{-\lambda i}} \\
& =e^{-\sum_{i=0}^{n} \log \left(1+\gamma_{k, 1} e^{\lambda_{k-1}\left(i-s_{i}\right)-\lambda i}+\gamma_{k, 2} e^{\lambda_{k+2} s_{i}-\lambda i}+(N-4) e^{-\lambda i}\right)} \\
& \geq e^{-\left(\sum_{i=0}^{n} \gamma_{k, 1} e^{\lambda_{k-1}\left(i-s_{i}\right)-\lambda i}+\gamma_{k, 2} e^{\lambda k+2 s_{i}-\lambda i}+c_{1} e^{-c_{2} i}\right)} \\
& \geq \delta e^{-\gamma_{k, 1} \sum_{i=0}^{n} e^{\lambda_{k-1}\left(i-s_{i}\right)-\lambda i}} e^{-\gamma_{k, 2} \sum_{i=0}^{n} e^{\lambda_{k+2} s_{i}-\lambda i}},
\end{aligned}
$$

for some $\delta>0$ not depending on the configuration $\mathbf{x}$. On the other hand, note that

$$
W_{n}\left(s_{1}, \ldots, s_{n}\right) \leq \prod_{i=0}^{n} \frac{1}{1+\gamma_{k, 1} e^{\lambda_{k-1}\left(i-s_{i}\right)-\lambda i}+\gamma_{k, 2} e^{\lambda_{k+2} s_{i}-\lambda i}} .
$$

The above inequalities yield the following lower and upper bounds

$$
\begin{aligned}
& \mathrm{P}_{\mathbf{x}}\left(A_{[1, n+1]}^{k, k+1}\right) \geq \delta \mathbb{E}_{p}\left(e^{-\gamma_{k, 1} \sum_{i=0}^{n} e^{\lambda_{k-1}\left(i-U_{i}\right)-\lambda i}} e^{-\gamma_{k, 2} \sum_{i=0}^{n} e^{\lambda_{k+2} U_{i}-\lambda i}}\right), \\
& \mathrm{P}_{\mathbf{x}}\left(A_{[1, n+1]}^{k, k+1}\right) \leq \mathbb{E}_{p}\left(\prod_{i=0}^{n} \frac{1}{1+\gamma_{k, 1} e^{\lambda_{k-1}\left(i-U_{i}\right)-\lambda i}+\gamma_{k, 2} e^{\lambda_{k+2} U_{i}-\lambda i}}\right) .
\end{aligned}
$$

We will also need a generalisation of lower bound (26) for probabilities $\mathrm{P}_{\mathbf{x}}\left(A_{[1, \tau]}^{k, k+1}\right)$, where $\tau$ is one of the following stopping times, $\min \left(n: S_{n}-c_{1} n \geq c_{2}\right), \min \left(n: n-S_{n} \geq c_{3}\right)$, and the minimum of two such stopping times. At the moment, we shall not further specify such stopping times as it will be clear later which one it refers to. Arguing similarly as in Eq. (22), one can obtain that

$$
\mathrm{P}_{\mathbf{x}}\left(A_{[1, \tau]}^{k, k+1}\right)=\sum_{n=0}^{\infty} \sum_{s(n)} p^{s_{n}} q^{n-s_{n}} W_{n}\left(s_{1}, \ldots, s_{n}\right) 1_{\left\{\mathrm{M}_{n}\right\}},
$$

where $\mathrm{M}_{n}$ is a set of paths $s(n)=\left(s_{1}, \ldots, s_{n}\right)$ for which $\tau=n+1$. Furthermore, similar to Eq. (23), we can rewrite equation above as

$$
\mathrm{P}_{\mathbf{x}}\left(A_{[1, \tau]}^{k, k+1}\right)=\mathbb{E}_{p}\left(W_{\tilde{\tau}}\left(U_{1}, \ldots, U_{\tilde{\tau}}\right)\right),
$$

where $\tilde{\tau}$ is a stopping time defined by replacing $S_{n}$ by $U_{n}$ in the same way as $\tau$ but in terms of random variables $U_{n}$. Proceeding similar to how we got lower bound (26) we obtain the following lower bound

$$
\mathrm{P}_{\mathbf{x}}\left(A_{[1, \tau]}^{k, k+1}\right) \geq \delta \mathbb{E}_{p}\left(e^{-\gamma_{k, 1} \sum_{i=0}^{\tilde{\tau}-1} e^{\lambda_{k-1}\left(i-U_{i}\right)-\lambda i}} e^{-\gamma_{k, 2} \sum_{i=0}^{\tilde{\tau}-1} e^{\lambda_{k+2} U_{i}-\lambda i}}\right) .
$$

Let us rewrite the lower bounds in terms of random sequences $\zeta_{1}, \zeta_{2}$ and $Z_{n}$ as defined in (12) and (13). In these notations, lower bounds (26) and (28) take the following form

$$
\mathrm{P}_{\mathbf{x}}\left(A_{[1, n+1]}^{k, k+1}\right) \geq \delta \mathbb{E}_{p}\left(e^{-\gamma_{k, 1} Z_{n}\left(\zeta_{1}\right)} e^{-\gamma_{k, 2} Z_{n}\left(\zeta_{2}\right)}\right)
$$

and

$$
\mathrm{P}_{\mathbf{x}}\left(A_{[1, \tau]}^{k, k+1}\right) \geq \delta \mathbb{E}_{p}\left(e^{-\gamma_{k, 1} Z_{\tilde{\tau}-1}\left(\zeta_{1}\right)} e^{-\gamma_{k, 2} Z_{\tilde{\tau}-1}\left(\zeta_{2}\right)}\right)
$$

respectively. 
Finally, letting $n \rightarrow \infty$ in (26) and (29) we obtain the following bound

$$
\begin{aligned}
\mathrm{P}_{\mathbf{x}}\left(A_{[1, \infty)}^{k, k+1}\right) & \geq \delta \mathbb{E}_{p}\left(e^{-\gamma_{k, 1} \sum_{i=0}^{\infty} e^{\lambda_{k-1}\left(i-U_{i}\right)-\lambda i}} e^{-\gamma_{k, 2} \sum_{i=0}^{\infty} e^{\lambda_{k+2} U_{i}-\lambda i}}\right) \\
& =\delta \mathbb{E}_{p}\left(e^{-\gamma_{k, 1} Z\left(\zeta_{1}\right)} e^{-\gamma_{k, 2} Z\left(\zeta_{2}\right)}\right) .
\end{aligned}
$$

\subsubsection{Proof of Lemma 4}

We start with the following proposition.

Proposition 8 Let $\mu_{p}$ be the Bernoulli measure defined in Sect. 4, and let $U_{n}, n \geq 1$, be the corresponding Binomial random variables [defined in (11)]. Then

(1) given $\varepsilon \in(0,1)$ and $\kappa>0$, there exist positive constants $c_{1}$ and $c_{2}$ such that

$$
\inf _{p \in(0,1)} \mu_{p}\left(\bigcap_{n=M}^{\infty}\left\{\frac{n}{2} p(1-\kappa)-c_{1} \leq U_{n} \leq n p(1+\kappa)+c_{2}\right\}\right) \geq \varepsilon,
$$

where $M=\left[p^{-1}\right]$ is the integer part of $p^{-1}$;

(2) given $\lambda>0$, there exists $\varepsilon_{1}>0$ such that

$$
\inf _{p \in(0,1)} \mathbb{E}_{p}\left(e^{-p \sum_{i=0}^{\infty} e^{-\lambda U_{i}}}\right) \geq \varepsilon_{1}
$$

Proof of Proposition 8 Set $U_{0}=0$ and define the following random variables

$$
\begin{aligned}
& V_{j}=U_{j M}-U_{(j-1) M}=\sum_{i=(j-1) M+1}^{j M} \xi_{i}, \quad j \geq 1, \\
& Y_{j}=V_{1}+\cdots+V_{j}, \quad j \geq 1, \\
& Y_{0}=0 .
\end{aligned}
$$

First, denote $a(p):=\mathbb{E}_{p}\left(V_{i}\right)=p M=p\left[p^{-1}\right]$ and note that $a(p) \in[1 / 2,1]$ for all $p \in(0,1)$. Moreover, $\operatorname{Var}\left(V_{i}\right)=\mathbb{E}_{p}\left(V_{i}^{2}\right)-\left(\mathbb{E}_{p}\left(V_{i}\right)\right)^{2}=p(1-p)\left[p^{-1}\right] \leq 1$. Now, consider the auxiliary process $\chi_{n}:=Y_{n}-n(1-\kappa) / 2+c^{\prime}$, with $\chi_{0}=c^{\prime}$. Note that $\mathbb{E}_{p}\left(\chi_{n+1}-\chi_{n} \mid \chi_{n}=\chi\right)=a(p)-(1-\kappa) / 2>0$. Moreover, if we define the stopping time $t_{x}=\min _{n \geq 0}\left\{\chi_{n}<x\right\}$, it follows from [11, Theorem 2.5.18] that there exist $x_{1}$ and $\alpha>0$ such that

$$
\mathbb{P}\left(\bigcap_{n=1}^{\infty}\left\{Y_{n} \geq \frac{n}{2}(1-\kappa)-\left(c^{\prime}-x_{1}\right)\right\}\right)=\mathbb{P}\left(t_{x_{1}}=\infty\right) \geq 1-\left(\frac{1+x_{1}}{1+\chi_{0}}\right)^{\alpha} .
$$

So, for every $\varepsilon \in(0,1)$ and $\kappa>0$, we can appropriately choose $\alpha$ and $\chi_{0}=c^{\prime}>x_{1}$ such that the probability in the above display is greater than $\varepsilon / 2$. Analogously, if we define $\chi_{n}=-Y_{n}+n(1+\kappa)$, the upper bound can be found exactly as above, yielding

$$
\mu_{p}\left(\bigcap_{n=1}^{\infty}\left\{\frac{n}{2}(1-\kappa)-c \leq Y_{n} \leq n(1+\kappa)+c\right\}\right) \geq \varepsilon
$$

Further, fix $n \geq M$. Let $m_{n}$ and $l_{n}$ be integers such that $n=m_{n} M+l_{n}$, where $l_{n}<M$. Then on event $\bigcap_{n=1}^{\infty}\left\{\frac{n}{2}(1-\kappa)-c \leq Y_{n} \leq n(1+\kappa)+c\right\}$ the following bounds hold

$$
U_{n} \geq Y_{m_{n}} \geq \frac{1}{2}\left(\frac{n}{M}-\frac{l_{n}}{M}\right)(1-\kappa)-c \geq \frac{1}{2} n p(1-\kappa)-c_{1},
$$


and

$$
U_{n} \leq Y_{m_{n}+1} \leq\left(\frac{n}{M}+\frac{M-l_{n}}{M}\right)(1+\kappa)+c \leq n p(1+\kappa)+c_{2} .
$$

Inequalities (33)-(35) yield bound (32).

Recall that $M=\left[p^{-1}\right]$, and so,

$$
p \sum_{i=0}^{M-1} e^{-\lambda U_{i}} \leq p M \leq 1 .
$$

By combining this bound with bound (32), it follows that given $\varepsilon \in(0,1)$ and $\kappa>0$ we can find $c_{1}>0$ such that with $\mu_{p}$-probability at least $\varepsilon$

$$
p \sum_{i=0}^{\infty} e^{-\lambda U_{i}} \leq 1+p \sum_{i=M}^{\infty} e^{-\lambda\left(\frac{1}{2} p i(1-\kappa)-c_{1}\right)} \leq C
$$

for some deterministic constant $C=C(\varepsilon, \lambda)$ and all $p \in(0,1)$. Therefore

$$
\inf _{p \in(0,1)} \mathbb{E}_{p}\left(e^{-p \sum_{i=0}^{\infty} e^{-\lambda U_{i}}}\right) \geq \varepsilon e^{-C}=\varepsilon_{1}>0,
$$

as required.

We are now ready to proceed with the proof of the lemma. Recall that $\lambda_{k}=\lambda_{k+1}=: \lambda$.

Proof of Part (1) of Lemma 4 Recall that in this case $\lambda_{k-1}<\lambda_{k}=\lambda_{k+1}=\lambda, \lambda \geq \lambda_{k+2}$ and $\Gamma_{k}=\max _{i} \Gamma_{i}$. Then,

$$
\gamma_{k, 1} Z\left(\zeta_{1}\right)=\gamma_{k, 1} \sum_{i=0}^{\infty} e^{\lambda_{k-1}\left(i-U_{i}\right)-\lambda i} \leq \sum_{i=0}^{\infty} e^{-\left(\lambda-\lambda_{k-1}\right) i} \leq C_{1}<\infty,
$$

where $C_{1}>0$ is a deterministic constant and we used that $\gamma_{k, 1} \leq 1$.

Further, if $\lambda>\lambda_{k+2}$, then

$$
\gamma_{k, 2} Z\left(\zeta_{2}\right)=\gamma_{k, 2} \sum_{i=0}^{\infty} e^{\lambda_{k+2} U_{i}-\lambda i} \leq \sum_{i=0}^{\infty} e^{-\left(\lambda-\lambda_{k+2}\right) i} \leq C_{2}<\infty,
$$

where $C_{2}>0$ is a deterministic constant and we used that $\gamma_{k, 2} \leq 1$. Then, using bounds (37) and (38) in lower bound (31) gives that $\mathrm{P}_{\mathbf{x}}\left(A_{[1, \infty)}^{k, k+1}\right) \geq \varepsilon$ for some $\varepsilon>0$, as claimed.

If $\lambda=\lambda_{k+2}$, then bound (38) cannot be used, and we proceed as follows. Note that in this case

$$
\gamma_{k, 2} Z\left(\zeta_{2}\right)=\gamma_{k, 2} \sum_{i=0}^{\infty} e^{\lambda\left(U_{i}-i\right)} \leq q \sum_{i=0}^{\infty} e^{\lambda\left(U_{i}-i\right)},
$$

as

$$
\gamma_{k, 2}=\frac{\Gamma_{k+2}}{\Gamma_{k}+\Gamma_{k+1}} \leq \frac{\Gamma_{k}}{\Gamma_{k}+\Gamma_{k+1}}=q=1-p,
$$

where $p$ is defined in (19). Further, combining bounds (37) and (39) in (31) we get that

$$
\mathrm{P}_{\mathbf{x}}\left(A_{[1, \infty)}^{k, k+1}\right) \geq \varepsilon_{1} \mathbb{E}_{p}\left(e^{-q \sum_{i=0}^{\infty} e^{\lambda\left(U_{i}-i\right)}}\right)=\varepsilon_{1} \mathbb{E}_{p}\left(e^{-p \sum_{i=0}^{\infty} e^{-\lambda U_{i}}}\right),
$$


where the equality holds by symmetry. It is left to note that the expectation in the right side of the last equation is bounded below uniformly over $p \in(0,1)$ by Part (2) of Proposition 8.

Proof of Part (2) of Lemma 4 Recall that in this case $\lambda_{k-1}=\lambda_{k}=\lambda_{k+1}=\lambda \geq \lambda_{k+2}$, $\Gamma_{k}=\max _{i} \Gamma_{i}$ and $\Gamma_{k-1} \leq \Gamma_{k+1}$. These conditions give that $e^{\lambda_{k-1}\left(i-U_{i}\right)-\lambda i}=e^{-\lambda U_{i}}$, $e^{\lambda_{k+2} U_{i}-\lambda i} \leq e^{\lambda\left(U_{i}-i\right)}$, and

$$
\gamma_{k, 1}=\frac{\Gamma_{k-1}}{\Gamma_{k}+\Gamma_{k+1}} \leq \frac{\Gamma_{k+1}}{\Gamma_{k}+\Gamma_{k+1}}=p .
$$

Recall also that $\gamma_{k, 2} \leq q=1-p$ [see (40)]. Using all these inequalities in lower bound (31) gives the following lower bound

$$
\mathrm{P}_{\mathbf{x}}\left(A_{[1, \infty)}^{k, k+1}\right) \geq \delta \mathbb{E}_{p}\left(e^{-p \sum_{i=0}^{\infty} e^{-\lambda U_{i}}} e^{-q \sum_{i=0}^{\infty} e^{\lambda\left(U_{i}-i\right)}}\right) .
$$

We have already shown in (36) that for any $\varepsilon \in(0,1)$ there exists constant $C=C(\varepsilon)>0$ such that

$$
\mu_{p}\left(p \sum_{i=0}^{\infty} e^{-\lambda U_{i}} \leq C\right) \geq \varepsilon \text { and } \mu_{p}\left(q \sum_{i=0}^{\infty} e^{\lambda\left(U_{i}-i\right)} \leq C\right) \geq \varepsilon
$$

for all $p$, where the second bound holds by symmetry. Choosing $\varepsilon>0.5$ we get that

$$
\mu_{p}\left(p \sum_{i=0}^{\infty} e^{-\lambda U_{i}} \leq C, q \sum_{i=0}^{\infty} e^{\lambda\left(U_{i}-i\right)} \leq C\right) \geq 2 \varepsilon-1>0,
$$

for all $p$. Combining this bound with Eq. (42) we finally obtain that $\mathrm{P}_{\mathbf{x}}\left(A_{[1, \infty)}^{k, k+1}\right) \geq \varepsilon_{2}$ for some $\varepsilon_{2}>0$, as claimed.

\subsubsection{Proof of Lemma 5}

Proof of Part (1) of Lemma 5 Note that at every time a particle is added to site $k$ or $k+1$, the allocation rates at these sites are multiplied by $e^{\lambda}$. In particular, if a particle is added to site $k$, then the allocation rate at $k-1$ is multiplied by $e^{\lambda_{k-1}}$. Otherwise, if a particle is added to site $k+1$, then the allocation rate at $k+2$ is multiplied by $e^{\lambda_{k+2}}$. Other rates remain unchanged. Thus, by allocating a particle at $k$ or $k+1$, the sum of rates at $k, k+1$ and $k+2$ over the sum of rates at all other sites is increased by a multiple constant. This yields the following exponential bound

$$
\mathrm{P}_{\mathbf{x}}\left(\bigcup_{i \neq k, k+1, k+2} A_{n+1}^{i} \mid A_{[1, n]}^{k, k+1}\right) \leq C_{1} e^{-C_{2} n},
$$

for some $C_{1}, C_{2}>0$. In turn, bound (44) implies that with a positive probability (not depending on $\mathbf{x}$ ) event $A_{[1, \infty)}^{k, k+1} \cup\left\{\tau_{k+2}<w_{k}^{+}\right\}$occurs as claimed. Note also that events $A_{[1, \infty)}^{k, k+1}$ and $\left\{\tau_{k+2}<w_{k}^{+}\right\}$are mutually exclusive. Thus, with a positive probability either all particles will be allocated at $k$ and $k+1$, or a particle is eventually placed at $k+2$ before anywhere else outside $k, k+1$ and $k+2$. Placing a particle at $k+2$ can violate condition (6) because the maximal allocating probability can be now attained at sites $k+2$ and $k+3$ as well. Part (1) of Lemma 5 is proved. 
Proof of Part (2) Lemma 5 Note that $e^{\lambda_{k-1}\left(i-U_{i}\right)-\lambda i}<e^{-\left(\lambda-\lambda_{k-1}\right) i}$ and $\lambda_{k-1}<\lambda$. Consequently, for any $n$

$$
Z_{n}\left(\zeta_{1}\right)=\sum_{i=0}^{n} e^{\lambda_{k-1}\left(i-U_{i}\right)-\lambda i} \leq \sum_{i=0}^{\infty} e^{-\left(\lambda-\lambda_{k-1}\right) i}<C<\infty .
$$

Note also that $\gamma_{k, 1} \leq 1$ and $\gamma_{k, 2} \leq 1$. Combining these inequalities with Eq. (45) and letting $n \rightarrow \infty$ in (29) gives that

$$
\mathrm{P}_{\mathbf{x}}\left(A_{[1, \infty]}^{k, k+1}\right) \geq \varepsilon_{1} \mathbb{E}_{p}\left(e^{-Z\left(\zeta_{2}\right)}\right)
$$

for some $\varepsilon_{1}>0$. Further, assumption $r<z_{2}$ implies that $\lambda_{k+2} p-\lambda<0$. Recall that parameter $r=x_{k+2}-x_{k-1}$ takes integer values, and $p=p(r)$ is a monotonically increasing function of $r$. Let $r_{0}$ be the maximal integer such that $r<z_{2}$ and $p_{0}=p\left(r_{0}\right)$, so that $\lambda_{k+2} p_{0}-\lambda<0$. It follows from Propositions 2 and 7 that for all $0<p<p_{0}$

$$
\mathbb{E}_{p}\left(e^{-Z\left(\zeta_{2}\right)}\right) \geq \mathbb{E}_{p_{0}}\left(e^{-Z\left(\zeta_{2}\right)}\right)>0,
$$

and, hence, $\mathrm{P}_{\mathbf{x}}\left(A_{[1, \infty]}^{k, k+1}\right) \geq \varepsilon$ for some uniform $\varepsilon>0$ over configurations $\mathbf{x}$ satisfying $r<z_{2}$. Part (2) of Lemma 5 is proved.

Proof of Part (3) of Lemma 5 We are going to use the following relaxation of upper bound (27)

$$
\mathrm{P}_{\mathbf{x}}\left(A_{[1, n+1]}^{k, k+1}\right) \leq \mathbb{E}_{p}\left(\prod_{i=0}^{n} \frac{1}{1+\gamma_{k, 2} e^{\lambda_{k+2} U_{i}-\lambda i}}\right) .
$$

Next, assumption $r \geq z_{2}$ implies that $\lambda_{k+2} p-\lambda \geq 0$. Therefore, by the strong law of large numbers, we get that $\mu_{p}$-a.s. $\lambda_{k+2} U_{i}-\lambda i \geq 0$ for infinitely many $i$ and, hence, $\prod_{i=0}^{n} \frac{1}{1+\gamma_{k, 2} e^{\lambda_{k+2} U_{i}-\lambda i}} \rightarrow 0$. The product is bounded by 1 , therefore, by the Lebesgue's dominated convergence theorem, the expectation in the right side of (46) tends to 0 as $n \rightarrow \infty$, which implies that

$$
\mathrm{P}_{\mathbf{x}}\left(A_{[1, \infty]}^{k, k+1}\right)=\lim _{n \rightarrow \infty} \mathrm{P}_{\mathbf{x}}\left(A_{[1, n+1]}^{k, k+1}\right)=0,
$$

as claimed. Note that Eq. (47) combined with Part (1) of the lemma further yields that $\mathrm{P}_{\mathbf{x}}\left(\tau_{k+2}<w_{k}^{+}\right)>\epsilon$ for some $\epsilon$.

Proof of Part (4) of Lemma 5 Define

$$
\widehat{n}=\min \left(n: \gamma_{k, 2} e^{\lambda_{k+2} S_{n}-\lambda n} \geq 1\right) .
$$

In other words, $\hat{n}$ is the first time when the allocation rate at site $k+2$ exceeds the sum of allocation rates at sites $k$ and $k+1$, becoming therefore, the maximal rate.

Applying lower bound (30) gives that

$$
\mathrm{P}_{\mathbf{x}}\left(A_{[1, \widehat{n}]}^{k, k+1}\right) \geq \delta \mathbb{E}_{p}\left(e^{-\gamma_{k, 1} Z_{\widehat{m}-1}\left(\zeta_{1}\right)} e^{-\gamma_{k, 2} Z_{\widehat{m}-1}\left(\zeta_{2}\right)}\right),
$$

where

$$
\widehat{m}=\min \left(m: \gamma_{k, 2} e^{\lambda_{k+2} U_{m}-\lambda m} \geq 1\right) .
$$


Equation(45) yields that $\gamma_{k, 1} Z_{\widehat{m}-1}\left(\zeta_{1}\right)<Z\left(\zeta_{1}\right)<C<\infty$. This allows us to rewrite bound (49) as follows $\mathrm{P}_{\mathbf{x}}\left(A_{[1, \widehat{n}]}^{k, k+1}\right) \geq \varepsilon_{2} \mathbb{E}_{p}\left(e^{-\gamma_{k, 2} Z_{\widehat{m}-1}\left(\zeta_{2}\right)}\right)$, for some $\varepsilon_{2}$. By assumption $r>z_{2}$. Let now $r_{0}$ be the minimal integer such that $r_{0}>z_{2}$ and $p_{0}=p\left(r_{0}\right)$. Then $\lambda_{k+2} p-\lambda>\lambda_{k+2} p_{0}-\lambda>0$ for any $p>p_{0}$. It follows from Propositions 6 and 7 that for all $p>p_{0}$

$$
\mathbb{E}_{p}\left(e^{-\gamma_{k, 2} Z_{\widehat{m}-1}\left(\zeta_{2}\right)}\right) \geq \mathbb{E}_{p_{0}}\left(e^{-Z\left(\eta_{2}\right)}\right)>0
$$

where $\eta_{2}$ is the sequence reciprocal to $\zeta_{2}$. Hence $\mathrm{P}_{\mathbf{x}}\left(A_{[1, \widehat{n}]}^{k, k+1}\right) \geq \varepsilon_{2} \epsilon_{2}>0$.

Next, recall event $B_{k}$ defined in (5). Note that $A_{[1, \widehat{n}]}^{k, k+1} \cap A_{\widehat{n}+1}^{k+2} \subseteq B_{k}$, so that $\mathrm{P}_{\mathbf{x}}\left(B_{k}\right) \geq$ $\varepsilon_{2} \epsilon_{2} / N>0$ as well.

It is left to show that the maximal rate $\max _{i} \Gamma_{i}$ relocates as described in (7). Clearly, this is always the case if $\lambda<\min \left(\lambda_{k+2}, \lambda_{k+3}\right)$. This might not be the case in the following particular situation. Namely, suppose that $\lambda_{k+3} \leq \lambda$ and initial configuration $\mathbf{x}$ is such that $\Gamma_{k}=\max _{i} \Gamma_{i}$ and $\Gamma_{k+3} e^{\lambda_{k+3}} \geq \Gamma_{k}$. In this case, if $\tau_{k+2}=1$, then the maximal rate might move to $k+3$. However, note that $\tau_{k+2} \geq 2$ on event $A_{[1, \widehat{n}]}^{k, k+1}$. Indeed, by definition (48) $\widehat{n} \geq 1$, and, hence, on this event $\tau_{k+2} \geq 2$ as $\tau_{k+2}>\widehat{n}$, so that at least one particle is deposited at $\{k, k+1\}$ by time $\tau_{k+2}$. It is not hard to check that placing one particle at $\{k, k+1\}$ makes impossible that relocation of $\max _{i} \Gamma_{i}$ to $k+3$ when $\lambda_{k+3} \leq \lambda$.

\subsubsection{Proof of Lemma 6}

First, note that the proof of Part (1) of Lemma 6 is analogous to the proof of Part (1) of Lemma 5 and we omit technical details. For simplicity of notation we denote $\lambda=\lambda_{k}=\lambda_{k+1}$ in the rest of the proof.

Proof of Part (2) of Lemma 6 Recall lower bound (31)

$$
\mathrm{P}_{\mathbf{x}}\left(A_{[1, \infty]}^{k, k+1}\right) \geq \delta \mathbb{E}_{p}\left(e^{-\gamma_{k, 1} Z\left(\zeta_{1}\right)} e^{-\gamma_{k, 2} Z\left(\zeta_{2}\right)}\right) .
$$

Note that $z_{1}<r<z_{2}$ if and only if both $\lambda_{k-1}(1-p)-\lambda<0$ and $\lambda_{k+2} p-\lambda<0$. Therefore, it follows from Proposition 2 that $\mu_{p}$-a.s. both $Z\left(\zeta_{1}\right)<\infty$ and $Z\left(\zeta_{2}\right)<\infty$. Consequently,

$$
\mathbb{E}_{p}\left(e^{-\gamma_{k, 1} Z\left(\zeta_{1}\right)} e^{-\gamma_{k, 2} Z\left(\zeta_{2}\right)}\right) \geq \mathbb{E}_{p}\left(e^{-Z\left(\zeta_{1}\right)} e^{-Z\left(\zeta_{2}\right)}\right) \geq \varepsilon(p)>0,
$$

as $\gamma_{k, i} \leq 1, i=1,2$, so that $\mathrm{P}_{\mathbf{x}}\left(A_{[1, \infty]}^{k, k+1}\right) \geq \delta \varepsilon(p)$. It is left to note that there is a finite number (depending only on $\lambda$ 's) of possible values of integer-valued parameter $r$ satisfying $z_{1}<r<z_{2}$, and, hence, the same number of possible values of probability $p$. Therefore, constant $\varepsilon(p)$ can be chosen as the minimal one for those values of $p$. This concludes the proof of the second part of the lemma.

Proof of Part (3) of Lemma 6 Let us start by noting the following. Assumption $r \leq z_{1}$ implies that $\lambda_{k-1}(1-p)-\lambda \geq 0$, and assumption $r \geq z_{2}$ implies that $\lambda_{k+2} p-\lambda \geq 0$. Therefore, the law of large numbers yields that $\mu_{p}$-a.s. at least one of the following events $\left\{\lambda_{k-1}\left(i-U_{i}\right)-\lambda i \geq 0\right\}$ and $\left\{\lambda_{k+2} U_{i}-\lambda i \geq 0\right\}$ occurs for infinitely many $i$. Consequently, $\mu_{p}$-a.s. $\prod_{i=0}^{n} \frac{1}{1+\gamma_{k, 1} e^{\lambda_{k-1}\left(i-U_{i}\right)-\lambda i}+\gamma_{k, 2} e^{\lambda_{k+2} U_{i}-\lambda i}} \rightarrow 0$, as $n \rightarrow \infty$. Using bound (27) and the Lebesgue dominated convergence theorem, we obtain that

$$
\mathrm{P}_{\mathbf{x}}\left(A_{[1, n+1]}^{k, k+1}\right) \leq \mathbb{E}_{p}\left(\prod_{i=0}^{n} \frac{1}{1+\gamma_{k, 1} e^{\lambda_{k-1}\left(i-U_{i}\right)-\lambda i}+\gamma_{k, 2} e^{\lambda_{k+2} U_{i}-\lambda i}}\right) \rightarrow 0,
$$


as $n \rightarrow \infty$. Hence, $\mathrm{P}_{\mathbf{x}}\left(A_{[1, \infty]}^{k, k+1}\right)=0$, and, hence, $\mathrm{P}_{\mathbf{x}}\left(D_{k}\right) \geq \varepsilon$, as claimed.

\subsubsection{Proof of Lemma 7}

The proof here is similar to the proof of Part (4) of Lemma 5. The common starting point is the lower bound (30) where $\tau$ and $\tilde{\tau}$ are appropriately chosen stopping times.

Proof of Parts (1) and (2) of Lemma 7 First, note that the random variables $Z\left(\zeta_{1}\right)$ and $Z\left(\zeta_{2}\right)$ are finite if $\lambda_{k-1}(1-p)-\lambda<0$ and $\lambda_{k+2} p-\lambda<0$, respectively. In fact, by our assumptions, precisely one of these conditions is necessarily satisfied so that one of $Z\left(\zeta_{1}\right)$ and $Z\left(\zeta_{2}\right)$ is almost surely finite. Then we apply bound (30) with the corresponding pair of stopping times $(\tau, \tilde{\tau})=\left(\widehat{n}_{2}, \widehat{m}_{2}\right)$ or $(\tau, \tilde{\tau})=\left(\widehat{n}_{1}, \widehat{m}_{1}\right)$ respectively, where

$$
\begin{aligned}
& \widehat{n}_{1}=\min \left(n: \gamma_{k, 1} e^{\lambda_{k-1}\left(n-S_{n}\right)-\lambda n} \geq 1\right), \\
& \widehat{n}_{2}=\min \left(n: \gamma_{k, 2} e^{\lambda_{k+2} S_{n}-\lambda n} \geq 1\right), \\
& \widehat{m}_{1}=\min \left(m: \gamma_{k, 1} e^{\lambda_{k-1}\left(m-U_{m}\right)-\lambda m} \geq 1\right), \\
& \widehat{m}_{2}=\min \left(m: \gamma_{k, 2} e^{\lambda_{k+2} U_{m}-\lambda m} \geq 1\right) .
\end{aligned}
$$

For concreteness, consider the case where $\{k, k+1\}$ is of type 2 and $r>z_{1} \geq z_{2}$, in which case $\lambda_{k-1}(1-p)-\lambda<0$ and $\lambda_{k+2} p-\lambda>0$. Applying bound (30) with $(\tau, \tilde{\tau})=\left(\widehat{n}_{2}, \widehat{m}_{2}\right)$ yields that

$$
\mathbf{P}_{\mathbf{x}}\left(A_{\left[1, \widehat{n}_{2}\right]}^{k, k+1}\right) \geq \delta \mathbb{E}_{p}\left(e^{-\gamma_{k, 1} Z_{\widehat{m}_{2}-1}\left(\zeta_{1}\right)} e^{-\gamma_{k, 2} Z_{\widehat{m}_{2}-1}\left(\zeta_{2}\right)}\right) .
$$

Condition $\lambda_{k-1}(1-p)-\lambda<0$ and Proposition 2 imply that $Z\left(\zeta_{1}\right)<\infty \mu_{p}$-a.s. Therefore, we can bound $\gamma_{k, 1} Z_{\widehat{m}_{2}-1}\left(\zeta_{1}\right) \leq Z\left(\zeta_{1}\right)$, as $\gamma_{k, 1} \leq 1$. Also, condition $\lambda_{k+2} p-\lambda>0$ and Proposition 5 imply that $\gamma_{k, 2} Z_{\widehat{m}_{2}-1}\left(\zeta_{2}\right)<\infty \mu_{p}$-a.s. Combining the above, we get to the following lower bound

$$
\mathrm{P}_{\mathbf{x}}\left(A_{\left[1, \widehat{n}_{2}\right]}^{k, k+1}\right) \geq \delta \mathbb{E}_{p}\left(e^{-Z\left(\zeta_{1}\right)} e^{-\gamma_{k, 2} Z_{\widehat{m}_{2}-1}\left(\zeta_{2}\right)}\right)
$$

Moreover, let $\eta_{2}$ be the sequence reciprocal to $\zeta_{2}$. Then, applying Proposition 5 again, we get that $Z\left(\eta_{2}\right)<\infty \mu_{p}$-a.s., $Z\left(\eta_{2}\right) \geq_{s t} \gamma Z_{\widehat{m}-1}\left(\zeta_{2}\right)$ and

$$
\mathbb{E}_{p}\left(e^{-Z\left(\zeta_{1}\right)} e^{-\gamma Z_{\widehat{m}-1}\left(\zeta_{2}\right)}\right) \geq \mathbb{E}_{p}\left(e^{-Z\left(\zeta_{1}\right)} e^{-Z\left(\eta_{2}\right)}\right)>0
$$

Let us show that, when $r>z_{1}$, the expectation in the right side of the preceding display is uniformly bounded below over $p=p(r)$. To this end, take the minimal integer $r_{0}$ such that $r_{0}>z_{1}$ so that condition $r>z_{1}$ implies $p>p_{0}=p\left(r_{0}\right)$, and, hence, $\lambda_{k-1}(1-p)-\lambda<$ $\lambda_{k-1}\left(1-p_{0}\right)-\lambda<0$ and $\lambda_{k+2} p-\lambda>\lambda_{k+2} p_{0}-\lambda>0$. This implies the following. First, consider the random variable $Z\left(\zeta_{1}\right)$ with distribution determined by parameter $p_{0}$. By Propositions 2 and 7, it follows that $Z\left(\zeta_{1}\right)$ is almost surely finite, and, moreover, it stochastically dominates any other random variable $Z\left(\zeta_{1}\right)$ with distribution determined by $p>p_{0}$. Second, consider the random variable $Z\left(\eta_{2}\right)$, where $\eta_{2}$ is a sequence reciprocal to sequence $\zeta_{2}$ whose distribution is determined by parameter $p_{0}$. By Propositions 2, 3 and 7, it follows that $Z\left(\eta_{2}\right)$ is almost surely finite and, moreover, it stochastically dominates any other random variable $Z\left(\eta_{2}\right)$, where $\eta_{2}$ is reciprocal to $\zeta_{2}$ whose distribution is determined by $p>p_{0}$. 
Therefore, $\mathbb{E}_{p}\left(e^{-Z\left(\zeta_{1}\right)} e^{-Z\left(\eta_{2}\right)}\right) \geq \mathbb{E}_{p_{0}}\left(e^{-Z\left(\zeta_{1}\right)} e^{-Z\left(\eta_{2}\right)}\right)$. Summarizing the above, we finally obtain that

$$
\mathrm{P}_{\mathbf{x}}\left(A_{\left[1, \widehat{n}_{2}\right]}^{k, k+1}\right) \geq \delta \mathbb{E}_{p_{0}}\left(e^{-Z\left(\zeta_{1}\right)} e^{-Z\left(\eta_{2}\right)}\right)>0 .
$$

We have considered here only the case where $\{k, k+1\}$ is of type 2 and $r>z_{1}$, but by rearranging the stopping times above, one should note that for all the remaining cases stated in Parts (1) and (2) of Lemma 7, the reasoning is exactly the same as above.

Proof of Part (3) of Lemma 7 Let us obtain the lower bound in Part (3) of Lemma 7. In this case $\{k, k+1\}$ is a local minimum of type 2 and $z_{2}<r<z_{1}$. The double inequality implies that both $\lambda_{k-1}(1-p)-\lambda>0$ and $\lambda_{k+2} p-\lambda>0$. As a result, both $Z\left(\zeta_{1}\right)$ and $Z\left(\zeta_{2}\right)$ are infinite. In this case we modify bound (30) with stopping times $\tau=\widehat{n}=\min \left(\widehat{n}_{1}, \widehat{n}_{2}\right)$ and $\tilde{\tau}=\widehat{m}=\min \left(\widehat{m}_{1}, \widehat{m}_{2}\right)$, as follows

$$
\begin{aligned}
P_{\mathbf{x}}\left(A_{[1, \widehat{n}]}^{k, k+1}\right) & \geq \delta \mathbb{E}_{p}\left(e^{-\gamma_{k, 1} Z_{\widehat{m}-1}\left(\zeta_{1}\right)} e^{-\gamma_{k, 2} Z_{\widehat{m}-1}\left(\zeta_{2}\right)}\right) \\
& \geq \delta \mathbb{E}_{p}\left(e^{-\gamma_{k, 1} Z_{\widehat{m}_{1}-1}\left(\zeta_{1}\right)} e^{-\gamma_{k, 2} Z_{\widehat{m}_{2}-1}\left(\zeta_{2}\right)}\right),
\end{aligned}
$$

where in the last inequality we bounded $\widehat{m}=\min \left(\widehat{m}_{1}, \widehat{m}_{2}\right)$ by $\widehat{m}_{1}$ and $\widehat{m}_{2}$, respectively. By Proposition $5 \mu_{p}$-a.s. both $\gamma_{k, 1} Z_{\widehat{m}_{1}-1}\left(\zeta_{1}\right)<\infty$ and $\gamma_{k, 2} Z_{\widehat{m}_{2}-1}\left(\zeta_{2}\right)<\infty$. Therefore, $\mathrm{P}_{\mathbf{x}}\left(A_{[1, \widehat{n}]}^{k, k+1}\right) \geq \varepsilon(p)>0$. Further, there are finitely many integers $r$ such that $z_{2}<r<z_{1}$. Consequently, there are finitely many corresponding values of probability $p$, and $\mathrm{P}_{\mathbf{x}}\left(A_{[1, \widehat{n}]}^{k, k+1}\right) \geq \varepsilon$ for some $\varepsilon>0$ uniformly over all values of $p$ in this finite set.

Finally, relocation of the maximal rate in all cases covered by Lemma 7 can be shown by modifying the argument used in the proof of Part (4) of Lemma 5.

\subsubsection{Proof of Lemma 8}

We skip proofs of Parts (1) and (3) as they are analogous to the proofs of Parts (1) and (3) of Lemma 5. Proofs of Parts (2) and (4) can be obtained by appropriately modifying proofs of Parts (2) and (4) of Lemma 5 and combining them with the ideas in the proof of Lemma 4. Modifications are due to condition $\lambda_{k-1}=\lambda$ implying that $z_{1}=-\infty<z_{2}$ (see Remark 2).

Proof of Part (2) of Lemma 8 Recall that in this case $r<z_{2}$, so that $\lambda_{k+2} p-\lambda<0$ and $p<p_{0}$, where $p_{0}$ is defined in Part (2) of Lemma 5. Repeating the proof of Part (2) of Lemma 5 and using that $\gamma_{k, 1} \leq p$ and $\gamma_{k, 2} \leq 1$ [see (41) and (40)] we obtain the following lower bound

$$
\mathrm{P}_{\mathbf{x}}\left(A_{[1, \infty]}^{k, k+1}\right) \geq \mathbb{E}_{p}\left(e^{-p Z\left(\zeta_{1}\right)} e^{-Z\left(\zeta_{2}\right)}\right)
$$

Our assumptions imply that both $Z\left(\zeta_{1}\right)$ and $Z\left(\zeta_{2}\right)$ are almost surely finite by Proposition 2 . Fix $\varepsilon>0.5$, let $C_{1}=C_{1}(\varepsilon)>0$ be such that

$$
\mu_{p}\left(p Z\left(\zeta_{1}\right) \leq C_{1}\right)=\mu_{p}\left(p \sum_{i=0}^{\infty} e^{-\lambda U_{i}} \leq C_{1}\right) \geq \varepsilon
$$

for all $p \in(0,1)$ [see (43)], and let $C_{2}=C_{2}(\varepsilon)$ be such that $\mu_{p_{0}}\left(Z\left(\zeta_{2}\right) \leq C_{2}\right) \geq \varepsilon$. The last inequality yields that $\mu_{p}\left(Z\left(\zeta_{2}\right) \leq C_{2}\right) \geq \mu_{p_{0}}\left(Z\left(\zeta_{2}\right) \leq C_{2}\right) \geq \varepsilon$, as $Z\left(\zeta_{2}\right)$, with distribution determined by parameter $p_{0}$, dominates any random variable $Z\left(\zeta_{2}\right)$ with distribution determined by parameter $p<p_{0}$. Finally, by using the same elementary argument 
as in the proof of Lemma 4 , we get that $\mu_{p}\left(p Z\left(\zeta_{1}\right) \leq C_{1}, Z\left(\zeta_{2}\right) \leq C_{2}\right) \geq 2 \varepsilon-1$, which implies that the expectation in the right side of (51) is bounded below away from zero, so that $\mathrm{P}_{\mathbf{x}}\left(A_{[1, \infty]}^{k, k+1}\right) \geq \varepsilon_{1}$ for some uniform $\varepsilon_{1}>0$ over configurations $\mathbf{x}$ satisfying $r<z_{2}$.

Proof of Part (4) of Lemma 8 Recall that in this case $r>z_{2}$, so that $\lambda_{k+2} p-\lambda>0$ and $p>p_{0}$, where $p_{0}$ is now defined in Part (4) of Lemma 5. Repeating the proof of Part (4) of Lemma 5 and using again that $\gamma_{k, 1} \leq p$ we obtain the following lower bound

$$
\mathrm{P}_{\mathbf{x}}\left(A_{[1, \widehat{n}]}^{k, k+1}\right) \geq \delta \mathbb{E}_{p}\left(e^{-p Z\left(\zeta_{1}\right)} e^{-\gamma_{k, 2} Z_{\widehat{m}-1}\left(\zeta_{2}\right)}\right),
$$

where $\widehat{n}$ and $\widehat{m}$ are defined in (48) and (50), respectively. Our assumptions imply that both $Z\left(\zeta_{1}\right)$ and $Z_{\widehat{m}-1}\left(\zeta_{2}\right)$ are almost surely finite by Propositions 2 and 5 . Further, Proposition 5 yields that

$$
\mathrm{P}_{\mathbf{x}}\left(A_{[1, \widehat{n}]}^{k, k+1}\right) \geq \delta \mathbb{E}_{p}\left(e^{-p Z\left(\zeta_{1}\right)} e^{-Z\left(\eta_{2}\right)}\right)
$$

where $\eta_{2}$ is the random sequence reciprocal to $\zeta_{2}$.

Let $\varepsilon>0.5$ and $C_{1}=C_{1}(\varepsilon)>0$ be such that (52) holds, and let $C_{2}=C_{2}(\varepsilon)$ be such that $\mu_{p_{0}}\left(Z\left(\eta_{2}\right) \leq C_{2}\right) \geq \varepsilon$. The last inequality yields that

$$
\mu_{p}\left(Z\left(\eta_{2}\right) \leq C_{2}\right) \geq \mu_{p_{0}}\left(Z\left(\eta_{2}\right) \leq C_{2}\right) \geq \varepsilon,
$$

as $Z\left(\eta_{2}\right)$, with distribution determined by parameter $p_{0}$, dominates any random variable $Z\left(\eta_{2}\right)$ with distribution determined by parameter $p>p_{0}$.

As at the same stage of the proof in Part (2) we can now conclude that the expectation in the right side of (53) is bounded below away from zero, which implies that $\mathrm{P}_{\mathbf{X}}\left(A_{[1, \widehat{n}]}^{k, k+1}\right) \geq \varepsilon_{2}$ for some uniform $\varepsilon_{2}>0$ over configurations $\mathbf{x}$ satisfying $r>z_{2}$.

\subsubsection{Proof of Corollary 1}

The critical cases where $r=z_{1}$ or $r=z_{2}$ need to be treated separately since these cases can not be proven directly by the above arguments. However, by a slight modification one can amend the proof of each lemma in order to encompass such critical cases.

The modification is the same for all lemmas, but for the sake of concreteness let us consider the critical case described in Part (3) of Lemma 5 assuming that $r=z_{2}$. We start by commenting on the same effect that we already discussed in the proof of Part (4) of Lemma 5. Namely, recall that if $\lambda_{k+3}<\lambda_{k}=\lambda_{k+1}, \Gamma_{k+3} e^{\lambda_{k+3}} \geq \Gamma_{k}$, and $\Gamma_{k}=\max _{i} \Gamma_{i}$, then $\tau_{k+2}=1$ makes the maximal rate move to $k+3$. One can check that the above situation is the only one that can possibly relocate the maximal rate to a site with smaller $\lambda$. In order to avoid such case, it is simply a matter of placing a particle at $k$ at the first step, which can be done with probability at least $1 / N$. Therefore, without loss of generality we can exclude this case.

Next, if at time $\tau_{k+2}$ the maximal rate relocates either to $k+2$, or to $k+3$ (provided $\left.\lambda_{k+3}>\lambda_{k}=\lambda_{k+1}\right)$ then we are done. Suppose the opposite, namely, that at time $\tau_{k+2}$ the maximal allocation rate remains where it was, that is, at $k$ or at $k+1$. It is left to note that given event $A_{\left[1, \tau_{k+2}-1\right]}^{k, k+1}$, placing a particle at site $k+2$ at moment $\tau_{k+2}$ increases the configuration parameter $r=x_{k+2}-x_{k-1}$ by 1 , so that the resulting configuration is such that $r>z_{2}$. By Part (4) of Lemma 5, the next allocated particles at $\{k, k+1\}$ will end up by relocating the maximal rate as prescribed.

Other critical cases can be handled similarly, and we skip straightforward technical details. 


\section{Proof of Theorem 1}

The idea of the proof goes briefly as follows. Given any initial state $X(0)=\mathbf{x}$, the site $k$ where $\Gamma_{k}(\mathbf{x})=\max _{i=1, \ldots, N}\left(\Gamma_{i}(\mathbf{x})\right)$ is identified. Then, a particle allocation strategy is drawn so that it always results in localization of growth as described in Theorem 1. Lemmas 1-8 enable us to identify the corresponding strategy for each particular case and bound its probability from below uniformly over initial configurations (see Remark 1). Should a particular strategy fail to happen, which means that at a certain step $n$ a particle is not allocated according to that strategy, but somewhere else, a new one is drawn and this procedure reiterates from $X(n)$. Since there is a finite number of possible strategies it follows from the renewal argument below that almost surely one of them eventually succeeds.

In what follows, when referring to Lemma 2 or one of Lemmas 4-8, this automatically includes the symmetric cases by re-labelling the graph in reverse order (as explained in Remark 3). Also, local minima of size 2 and type 1 automatically include the limiting case described in Remark 2.

Let $X(n)=\mathbf{x}$ be a fixed and arbitrary configuration, and:

(1) Assume that $\Gamma_{k}(\mathbf{x})=\max _{i=1, \ldots, N}\left(\Gamma_{i}(\mathbf{x})\right)$ and $\lambda_{k-1} \neq \lambda_{k} \neq \lambda_{k+1}$.

(1.1) Let $k$ be a local maximum. By Lemma 1, with positive probability, all subsequent particles are allocated at $k$.

(1.2) Let $k$ be either a growth point, or a local minimum. By Lemmas 2 and 3 , with positive probability, the maximal rate relocates in finite time to one of its nearest neighbours having parameter $\lambda>\lambda_{k}$.

(2) Assume that $\Gamma_{k}(\mathbf{x})=\max _{i=1, \ldots, N}\left(\Gamma_{i}(\mathbf{x})\right)$ and that additional assumptions of Lemma 4 are satisfied. Lemma 4 yields that, with positive probability, all subsequent particles are allocated at sites $\{k, k+1\}$.

(3) Assume that $\max \left(\Gamma_{k}(\mathbf{x}), \Gamma_{k+1}(\mathbf{x})\right)=\max _{i} \Gamma_{i}(\mathbf{x})$, where $\{k, k+1\}$ is either a saddle point, or a local minimum of size 2 and type 1 . Additional assumptions on $\mathbf{x}$, as described in Part (2) of Lemmas 5, 6 and 8, guarantee that, with positive probability, all subsequent particles are allocated at sites $\{k, k+1\}$.

(4) Assume that $\max \left(\Gamma_{k}(\mathbf{x}), \Gamma_{k+1}(\mathbf{x})\right)=\max _{i} \Gamma_{i}(\mathbf{x})$, where $\{k, k+1\}$ is either a saddle point of size 2, or a local minimum of size 2 of either type. Assume also that configuration $\mathbf{x}$ is such that assumptions as in the preceding item do not hold. Such cases are covered by Lemmas: 5, Parts (3) and (4), 6 Part (3), 7 and 8, and finally, 8 Parts (3) and (4) complemented by Corollary 1 . In all those cases, with positive probability, the maximal rate eventually relocates in a random but finite time to a site with larger parameter $\lambda$.

(5) Finally, for the remaining cases of local minima, maxima or saddle points of size greater than 2, it is not hard to check that such cases can be reduced to one, or a combination, of the above items.

Thus, for every configuration $\mathbf{x}$ and every set of positive real parameters $\Lambda=\left(\lambda_{k}\right)_{k=1}^{N}$, we have identified two types of events. First, there are events resulting in localisation of growth at either a single site or a pair of neighbouring sites [as described in Theorem 1 Parts (1) and (2), respectively]. Call such events L-events. Second, there are events resulting in relocation of the maximal rate. Call such events R-events.

The next step of the proof is to define a sequence of random moments of time $\left(T_{j}\right)_{j \geq 0}$ called renewal moments. First, set $T_{0}=0$. Now, given $T_{j}$, let us define $T_{j+1}$. Suppose that at time $T_{j}$ the process is at state $\mathbf{x}$. We identify an event $R_{1}, \ldots, R_{m} L$ (strategy) formed by 
a sequence of $m \mathrm{R}$-events (possibly none) ending at an L-event. At the fist moment of time $t>T_{j}$ a particle is not allocated according to $R_{1}, \ldots, R_{m} L$, we set $T_{j+1}=t$.

Note that $\mathrm{R}$-events are defined in a way so that the maximal rate always relocates to a site with strictly larger parameter $\lambda$. It follows that the number of R-events preceding any L-event is bounded by the number of different values of $\lambda_{i}, i=1, \ldots, N$. Then, by Lemmas 18 , probabilities of events $R_{1}, \ldots, R_{m} L$ are bounded below uniformly over configurations, where $m \leq N$.

Further, let $j_{\max }:=\max \left\{j \geq 0: T_{j}<\infty\right\}$. Lemmas $1-8$ imply the existence of an uniform bound $\epsilon>0$ such that $\mathrm{P}\left(T_{j}=\infty\right) \geq \epsilon$ on $\left\{T_{j-1}<\infty\right\}$. Therefore, $\mathrm{P}\left(T_{j}<\right.$ $\infty) \leq 1-\epsilon$ on $\left\{T_{j-1}<\infty\right\}$, or equivalently, $\mathrm{P}\left(j_{\max } \geq j \mid j_{\max } \geq j-1\right)<1-\epsilon$. Thus, $\mathrm{P}\left(j_{\max }<\infty\right)=1$. This implies that $T_{j}=\infty$ for some $j$, so that, with probability one, a certain allocation strategy $R_{1}, \ldots, R_{m} L$ eventually succeeds, that is the growth process localises as claimed.

Finally, the long term behaviour of ratio $X_{k+1}(n) / X_{k}(n)$ described in item (ii) of the theorem is implied by the law of large numbers for the Binomial distribution. This follows straightforwardly from the proofs of Lemma 4 and Parts (2) of Lemmas 5, 6 and 8. The theorem is proved.

Acknowledgements M.C.'s research is supported by CNPq (Grant Number 248679/2013-9). M.V.'s research is supported by CNPq (Grant Numbers 305369/2016-4 and 302593/2013-6). V.S.'s research is partially supported by LMS (Grant Number 41562). The authors are grateful to Andrew Wade for suggestions that improved the presentation.

Open Access This article is distributed under the terms of the Creative Commons Attribution 4.0 International License (http://creativecommons.org/licenses/by/4.0/), which permits unrestricted use, distribution, and reproduction in any medium, provided you give appropriate credit to the original author(s) and the source, provide a link to the Creative Commons license, and indicate if changes were made.

\section{References}

1. Barabási, A.L., Stanley, H.E.: Fractal Concepts in Surface Growth. Cambridge University Press, Cambridge (1995). https://doi.org/10.1017/CBO9780511599798

2. Bartelt, M.C., Privman, V.: Kinetics of irreversible monolayer and multilayer adsorption. Int. J. Mod. Phys. B 5(18), 2883-2907 (1991). https://doi.org/10.1142/S0217979291001127

3. Benaïm, M., Benjamini, I., Chen, J., Lima, Y.: A generalized Pólya's urn with graph based interactions. Random Struct. Algorithms 46(4), 614-634 (2015). https://doi.org/10.1002/rsa.20523

4. Collevecchio, A., Cotar, C., LiCalzi, M.: On a preferential attachment and generalized Pólya's urn model. Ann. Appl. Probab. 23(3), 1219-1253 (2013). https://doi.org/10.1214/12-AAP869

5. Davis, B.: Reinforced random walk. Probab. Theory Relat. Fields 84(2), 203-229 (1990). https://doi.org/ 10.1007/BF01197845

6. Eden, M.: A two-dimensional growth process. In: Proceedings of 4th Berkeley Symposium on Mathematical Statistics and Probability, vol. IV, pp. 223-239. University of California Press, Berkeley (1961)

7. Evans, J.: Random and cooperative sequential adsorption. Rev. Mod. Phys. 65, 1281-1329 (1993)

8. Evans, J.W.: Chap. 10: random and cooperative sequential adsorption: exactly solvable models on 1D lattices, continuum limits, and 2D extensions. In: Privman, V. (ed.) Nonequilibrium Statistical Mechanics in One Dimension, pp. 205-228. Cambridge Press, Cambridge (1997)

9. Fleurke, S.R., Formentin, M., Külske, C.: Dependent particle deposition on a graph: concentration properties of the height profile. Markov Process. Relat. Fields 17(2), 187-208 (2011)

10. Jullien, R., Meakin, P.: Simple three-dimensional models for ballistic deposition with restructuring. Europhys. Lett. 4, 1385-1390 (1987)

11. Menshikov, M., Popov, S., Wade, A.: Non-homogeneous Random Walks-Lyapunov Function Methods for Near-Critical Stochastic Systems. No. 209 in Cambridge Tracts in Mathematics. Cambridge Press, Cambridge (2017) 
12. Oliveira, R.I.: The onset of dominance in balls-in-bins processes with feedback. Random Struct. Algorithms 34(4), 454-477 (2009). https://doi.org/10.1002/rsa.20261

13. Pemantle, R.: A survey of random processes with reinforcement. Probab. Surv. 4, 1-79 (2007). https:// doi.org/10.1214/07-PS094

14. Penrose, M.D.: Existence and spatial limit theorems for lattice and continuum particle systems. Probab. Surv. 5, 1-36 (2008). https://doi.org/10.1214/07-PS112

15. Penrose, M.D.: Growth and roughness of the interface for ballistic deposition. J. Stat. Phys. 131(2), 247-268 (2008). https://doi.org/10.1007/s10955-008-9507-1

16. Penrose, M.D., Yukich, J.E.: Limit theory for random sequential packing and deposition. Ann. Appl. Probab. 12(1), 272-301 (2002). https://doi.org/10.1214/aoap/1015961164

17. Richardson, D.: Random growth in a tessellation. Proc. Camb. Philos. Soc. 74, 515-528 (1973)

18. Schürger, K.: On the asymptotic geometrical behaviour of a class of contact interaction processes with a monotone infection rate. Z. Wahrsch. Verwandte Gebiete 48(1), 35-48 (1979). https://doi.org/10.1007/ BF00534880

19. Shcherbakov, V., Volkov, S.: Stability of a growth process generated by monomer filling with nearestneighbour cooperative effects. Stoch. Process. Appl. 120(6), 926-948 (2010). https://doi.org/10.1016/j. spa.2010.01.020

20. Strassen, V.: The existence of probability measures with given marginals. Ann. Math. Stat. 36, 423-439 (1965). https://doi.org/10.1214/aoms/1177700153

21. Thorisson, H.: Coupling, Stationarity, and Regeneration. Probability and Its Applications. Springer, New York (2000). https://doi.org/10.1007/978-1-4612-1236-2

22. Witten Jr., T.A., Sander, L.M.: Diffusion-limited aggregation, a kinetic critical phenomenon. Phys. Rev. Lett. 47, 1400-1403 (1981) 